\title{
Multi-variable, multi-configuration testing of ORCHIDEE land surface model water flux and storage estimates across semi-arid sites in the southwestern US
}

5 Natasha MacBean1*, Russell L. Scott2, Joel A. Biederman2, Catherine Ottlé3, Nicolas Vuichard3, Agnès Ducharne4, Thomas Kolb5, Sabina Dore6, Marcy Litvak7, David J.P. Moore8.

1Department of Geography, Indiana University, Bloomington, IN 47405, USA.

2Southwest Watershed Research Center, United States Agricultural Department, Agricultural Research Service, Tucson, AZ 85719, USA.

10 3Laboratoire des Sciences du Climat et de l'Environnement, LSCE/IPSL, CEA-CNRS-UVSQ, Université Paris-Saclay, Gifsur-Yvette, F-91191, France.

${ }_{4}$ UMR METIS, Sorbonne Université, CNRS, EPHE, Paris, F-75005, France

5School of Forestry, Northern Arizona University, Flagstaff, AZ, 86011, USA.

6Hydrofocus, Inc., Davis, CA, 95618, USA.

157 Department of Biology, University of New Mexico, Albuquerque, NM, 87131, USA.

${ }_{8}$ School of Natural Resources and the Environment, University of Arizona, Tucson, AZ, 85721, USA.

*Correspondence to: Natasha MacBean (nlmacbean@gmail.com)

Table S1: Depths of the ORCHIDEE 11-layer discretized hydrology model

\begin{tabular}{|c|c|c|}
\hline ORCHIDEE Layer & Layer thickness (m) & Cumulative Depth (m) \\
\hline $\mathbf{1}$ & 0.001 & 0.001 \\
\hline $\mathbf{2}$ & 0.003 & 0.004 \\
\hline $\mathbf{3}$ & 0.006 & 0.01 \\
\hline $\mathbf{4}$ & 0.012 & 0.022 \\
\hline $\mathbf{5}$ & 0.023 & 0.045 \\
\hline $\mathbf{6}$ & 0.047 & 0.092 \\
\hline $\mathbf{7}$ & 0.092 & 0.186 \\
\hline $\mathbf{8}$ & 0.188 & 0.374 \\
\hline $\mathbf{9}$ & 0.375 & 0.750 \\
\hline $\mathbf{1 0}$ & 0.750 & 1.5 \\
\hline $\mathbf{1 1}$ & 0.5 & 2.0 \\
\hline
\end{tabular}


Figure S1: Complete daily time series comparing the 2LAY (green curve) and 11LAY (blue curve) simulations for the following hydrological variables: i) ET (top panel for each site) compared to observations (black curve); ii) surface runoff (2nd panel for each site); iii) drainage (3rd panel for each site); and iv) total $2 \mathrm{~m}$ column volumetric water content (VWC) soil moisture (bottom panel for each site). Precipitation is shown in the grey bars in the bottom panel for each site. Sites in following order: a) US-Fuf; b) US-Vcp;

a) US-Fuf
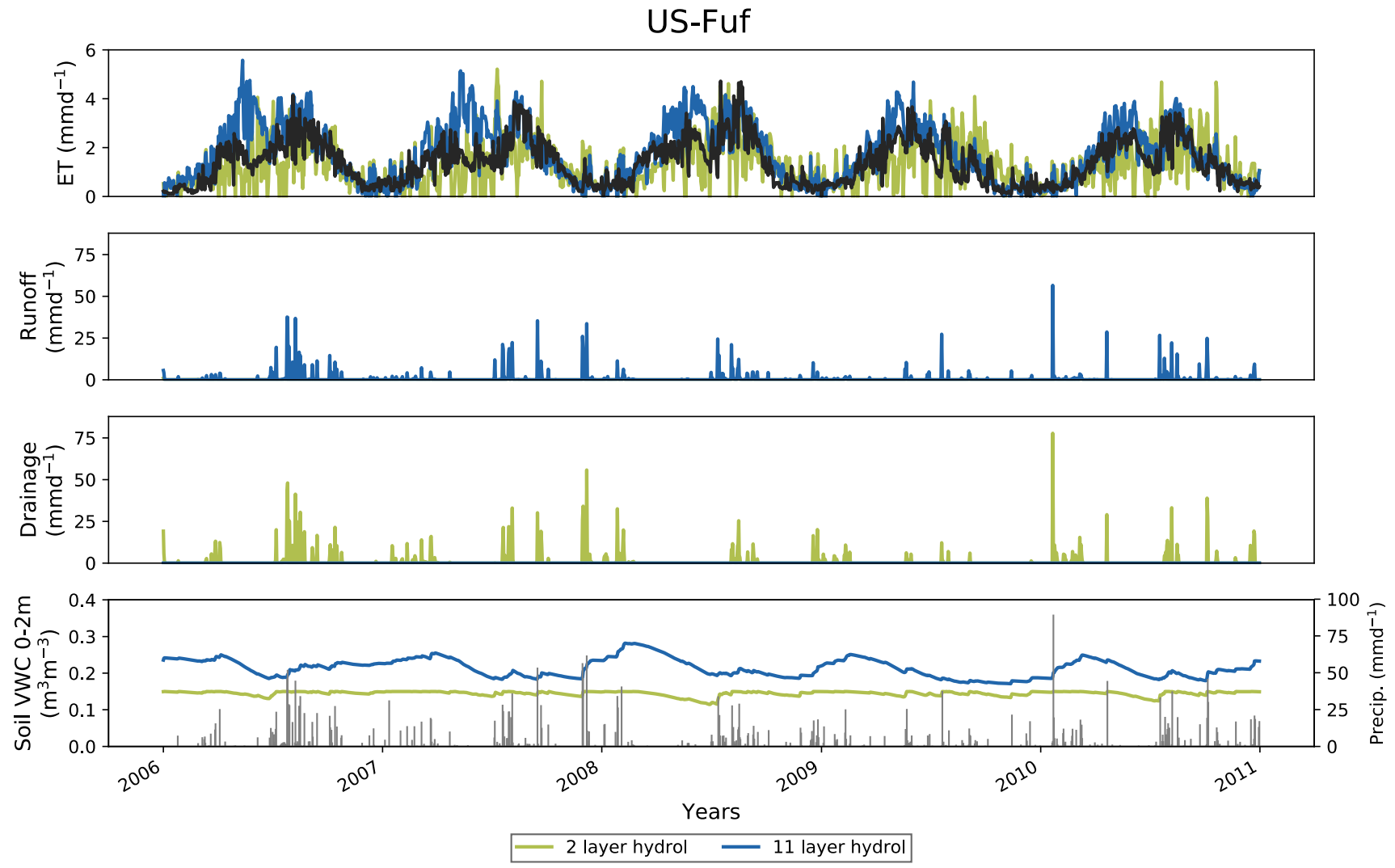
b) US-Vep
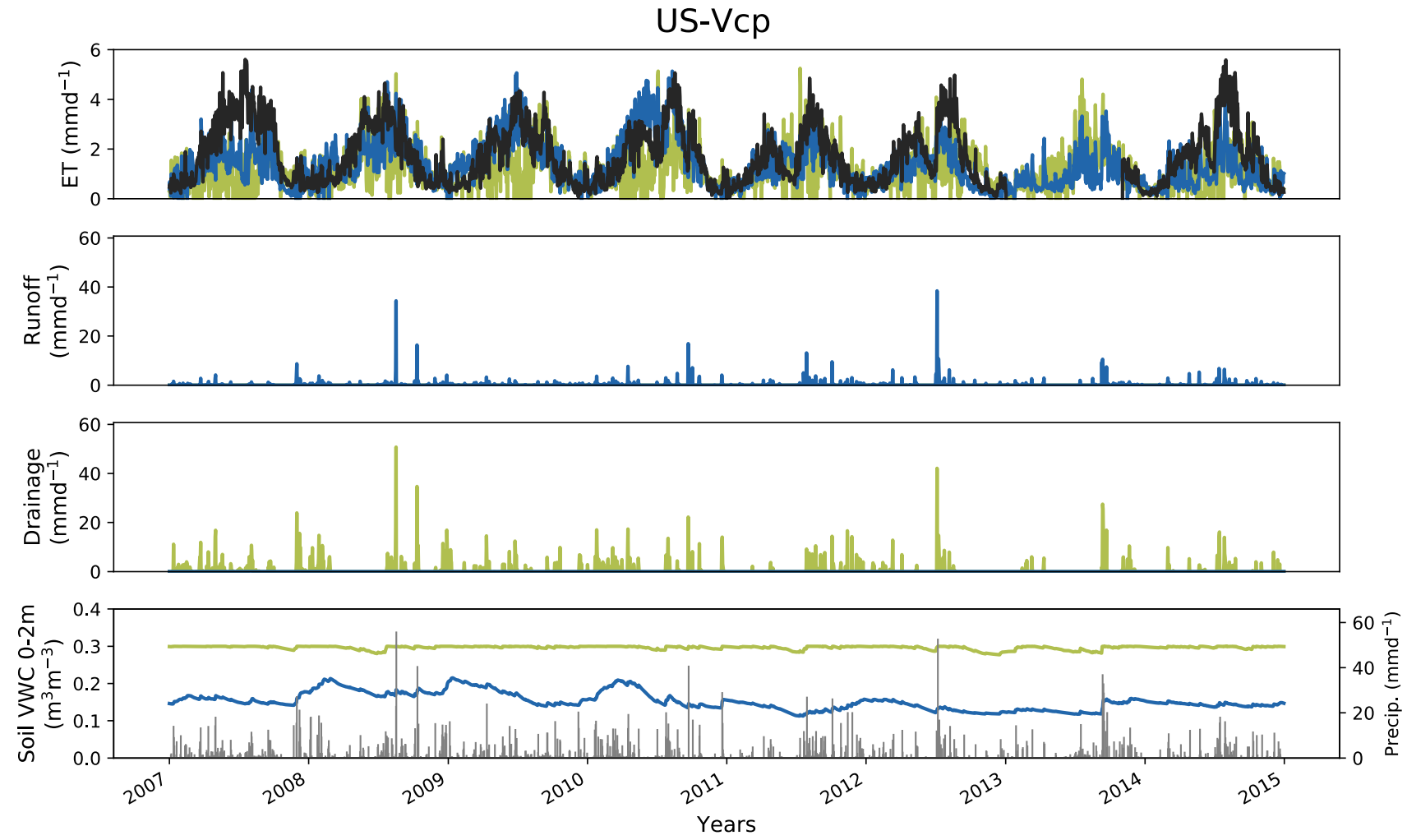
US-SRM
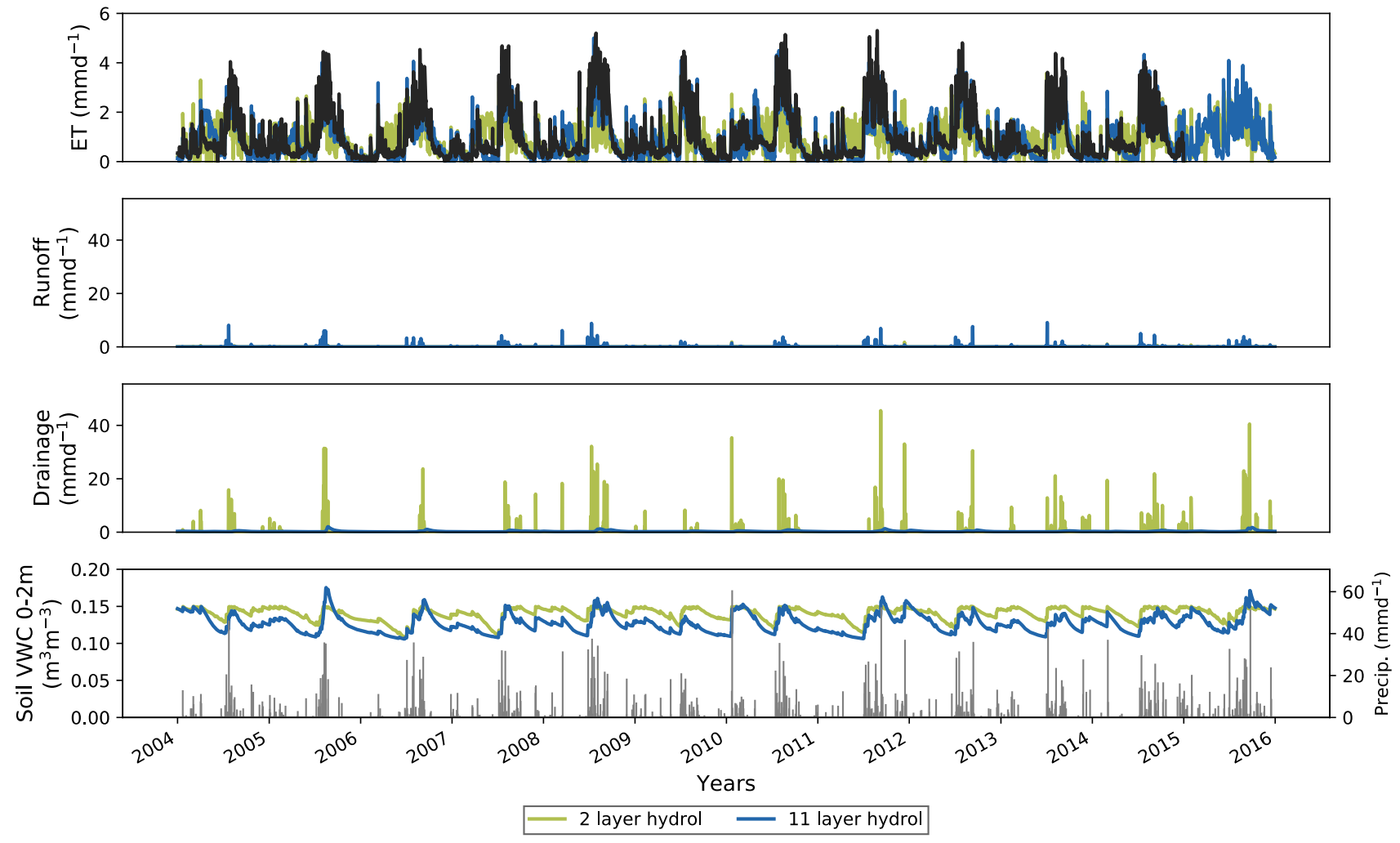
d) US-Whs

US-Whs
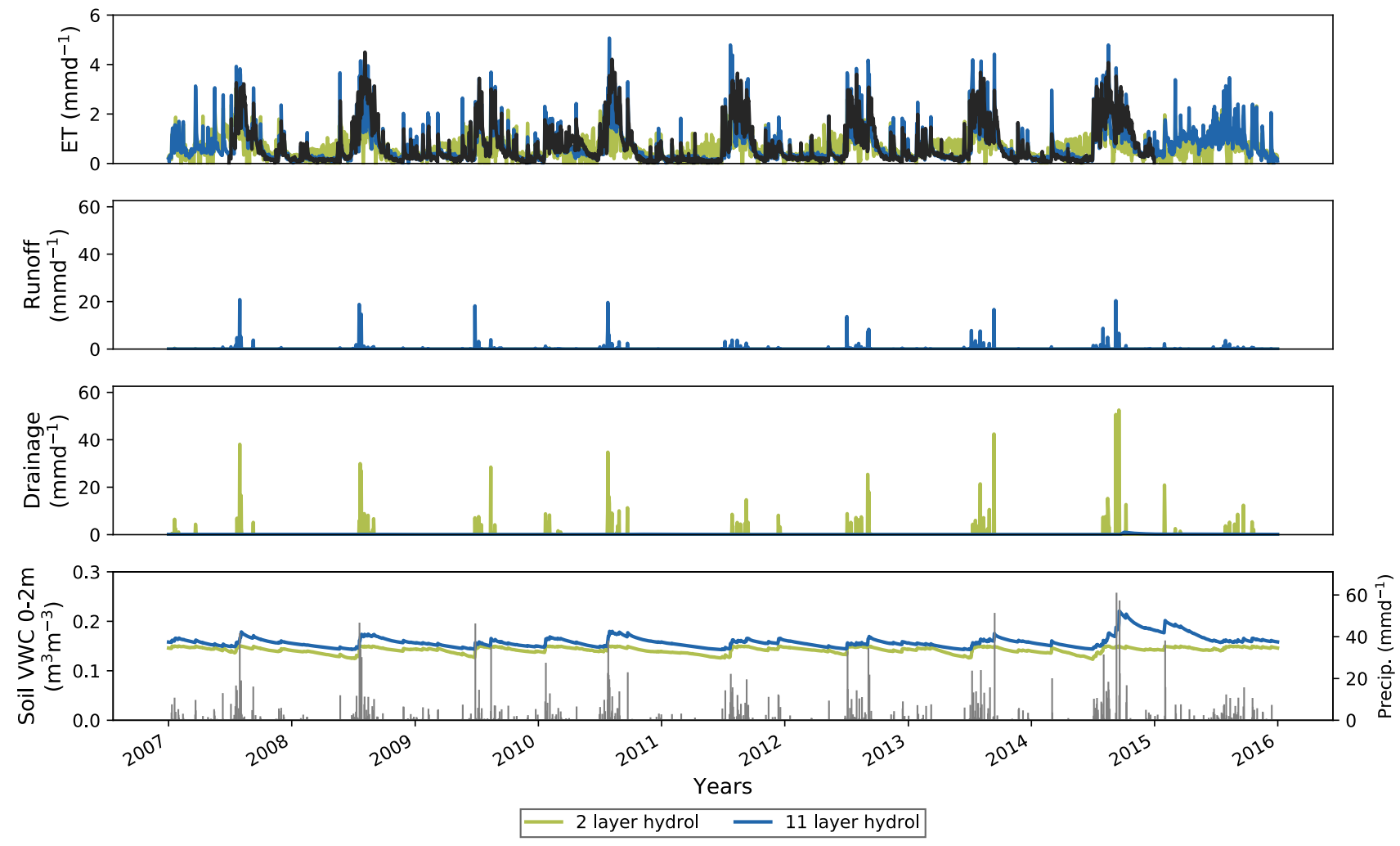
e) US-SRG

US-SRG
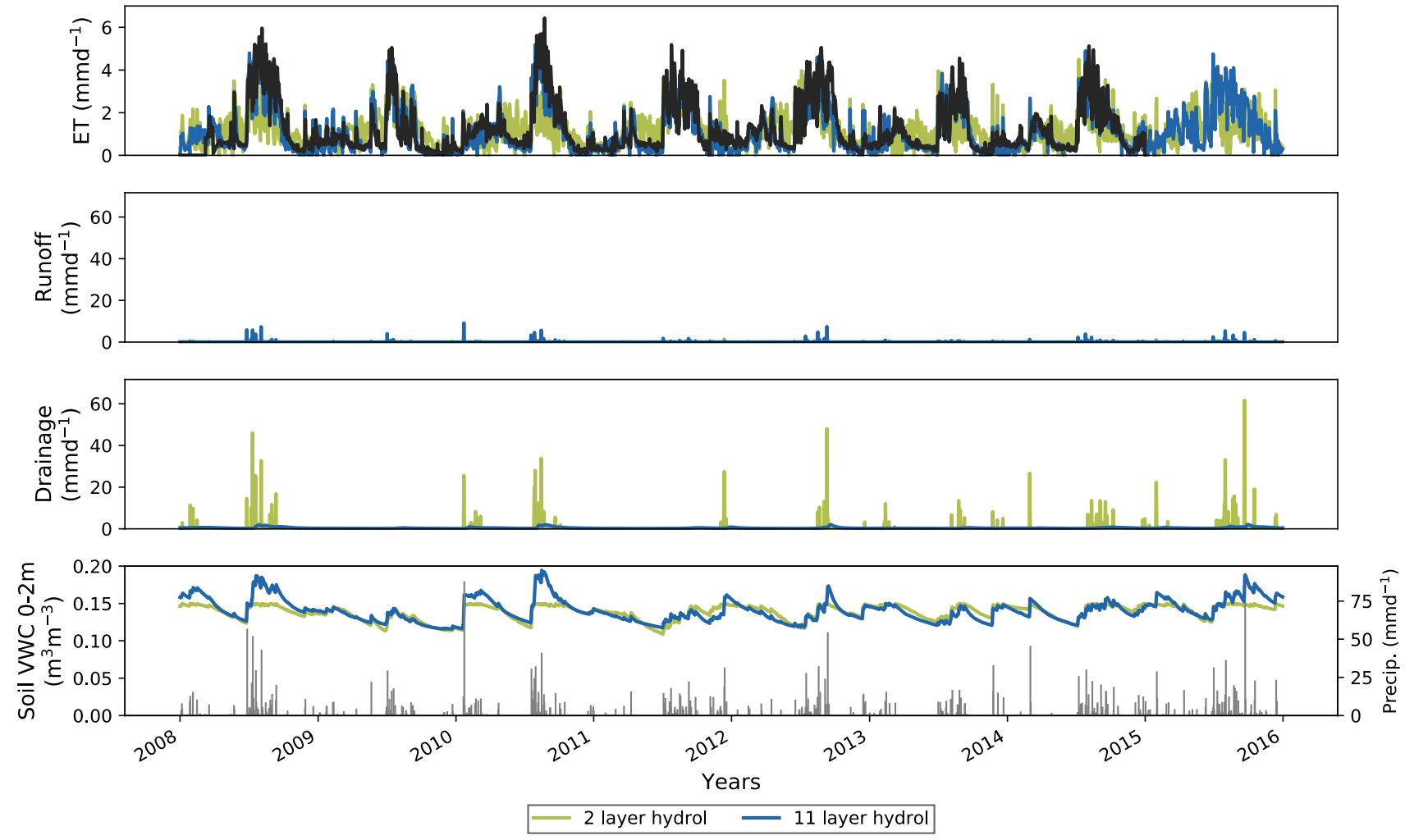


\section{f) US-W kg}
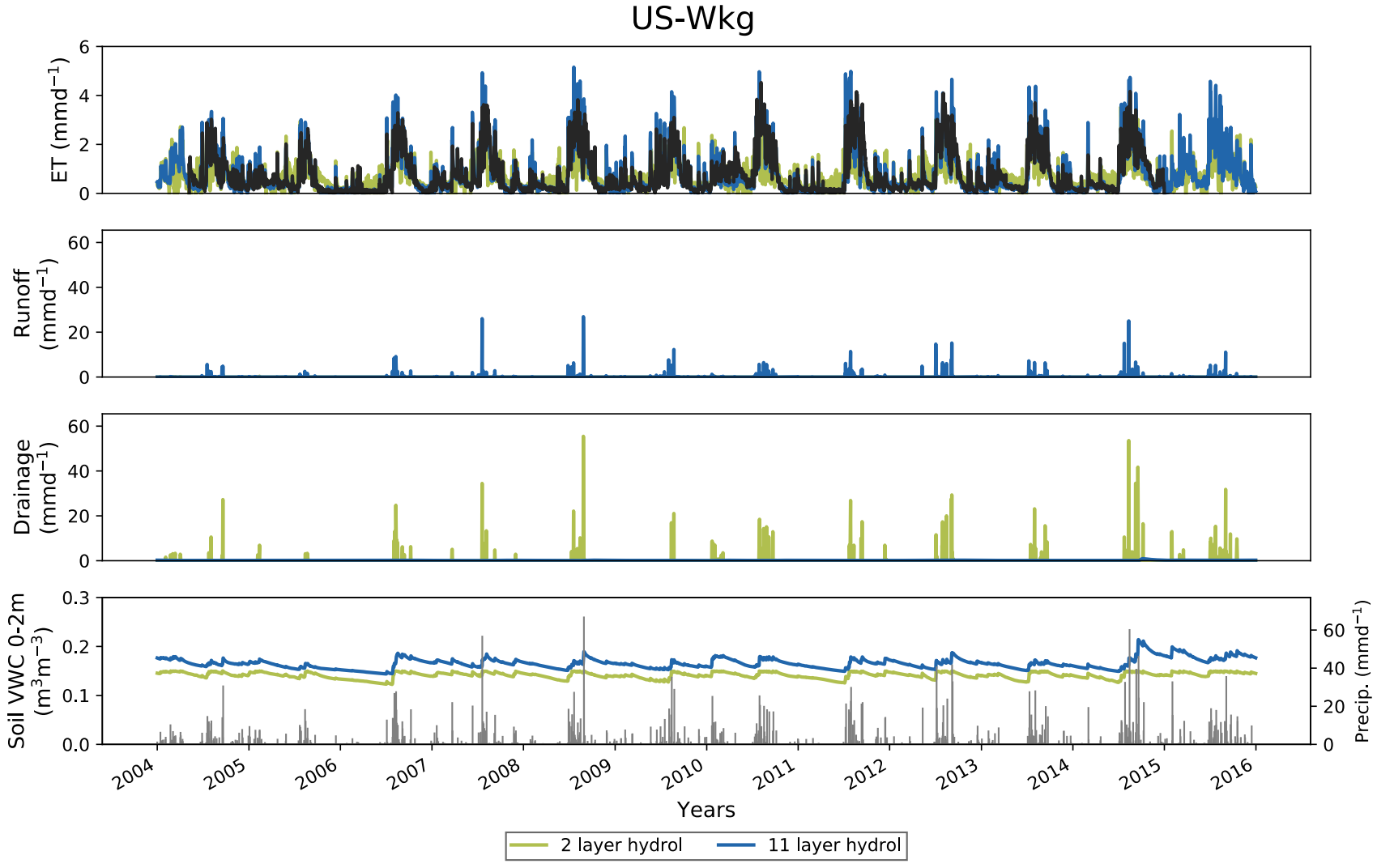

110 
Figure S2: Complete daily time series of variables influencing changes in ET between the 2LAY (green curve) and 11LAY (blue

curve) simulations at three sites: left column = high elevation tree-dominated site (US-Fuf); middle column = low elevation mesquite shrub-dominated site (US-SRM); right column = low elevation C4 grass site (US-SRG). At each site, top panel: LAI; 2nd panel: ET compared to observations (black curve); 3rd panel: transpiration; 4th panel: bare soil evaporation; 5th panel: empirical water limitation function $(\beta)$ that scales photosynthesis and stomatal conductance; bottom panel: soil moisture expressed as volumetric water content (VWC) in the uppermost $10 \mathrm{~cm}$ of the soil. Precipitation is shown in the grey bars in the bottom panel for each site.

130 Sites in following order: a) US-Fuf; b) US-Vcp; c) US-SRM; d) US-Whs; e) US-SRG; f) US-Wkg. Precipitation is shown in the grey lines in the bottom panel for each site.

a) US-Fuf
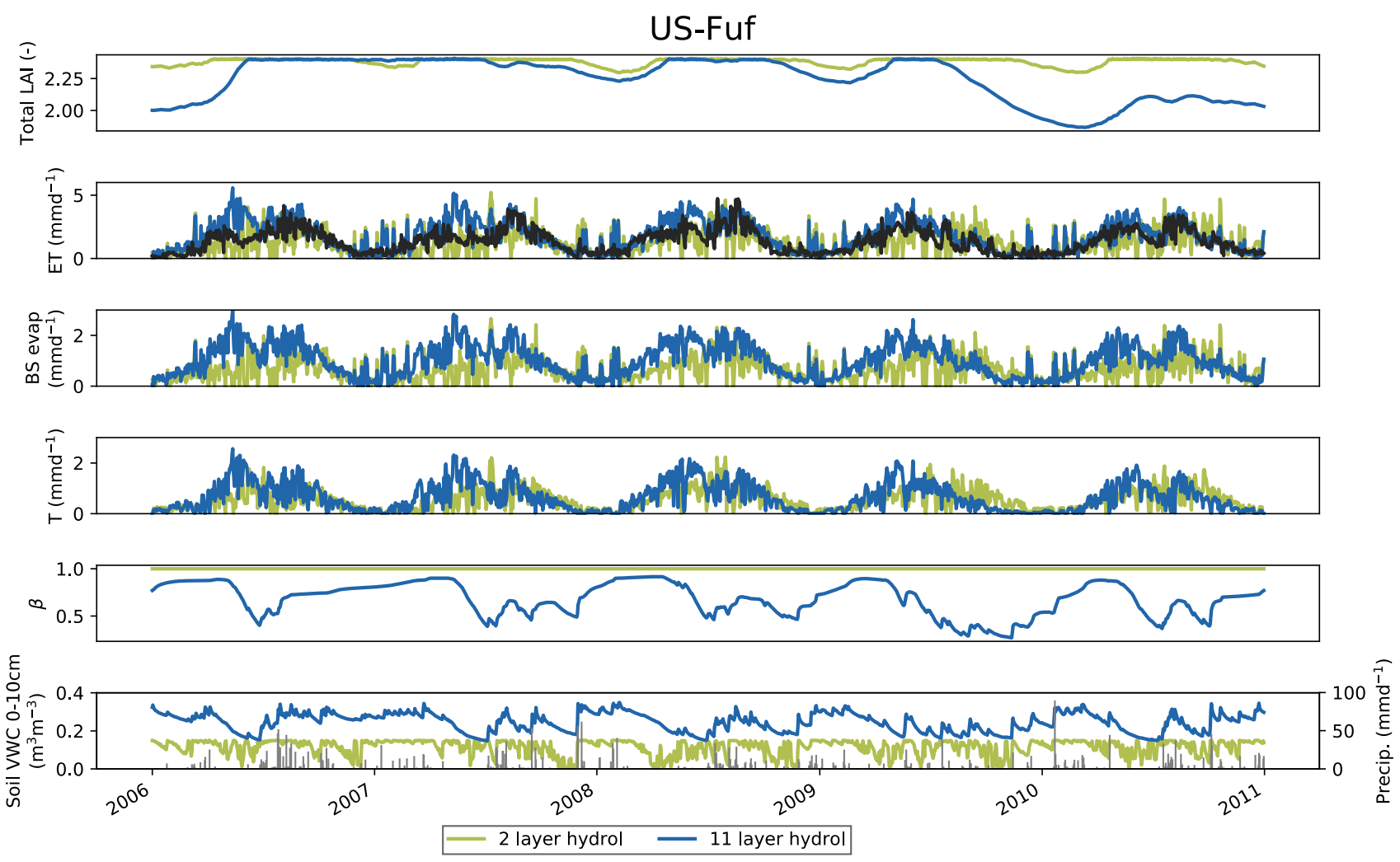
b) US-Vcp

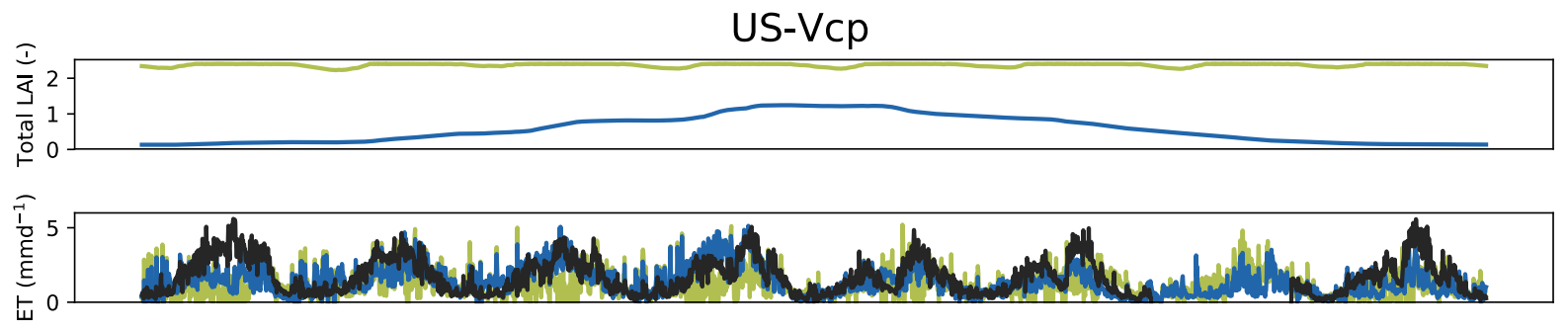

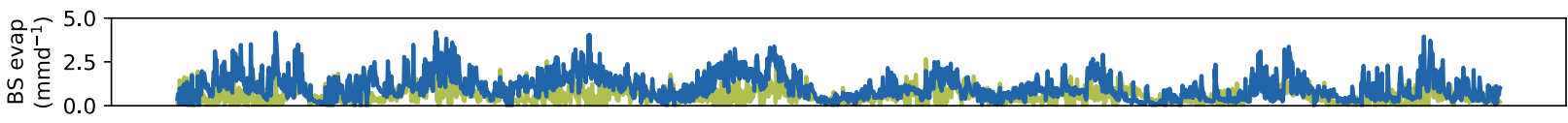
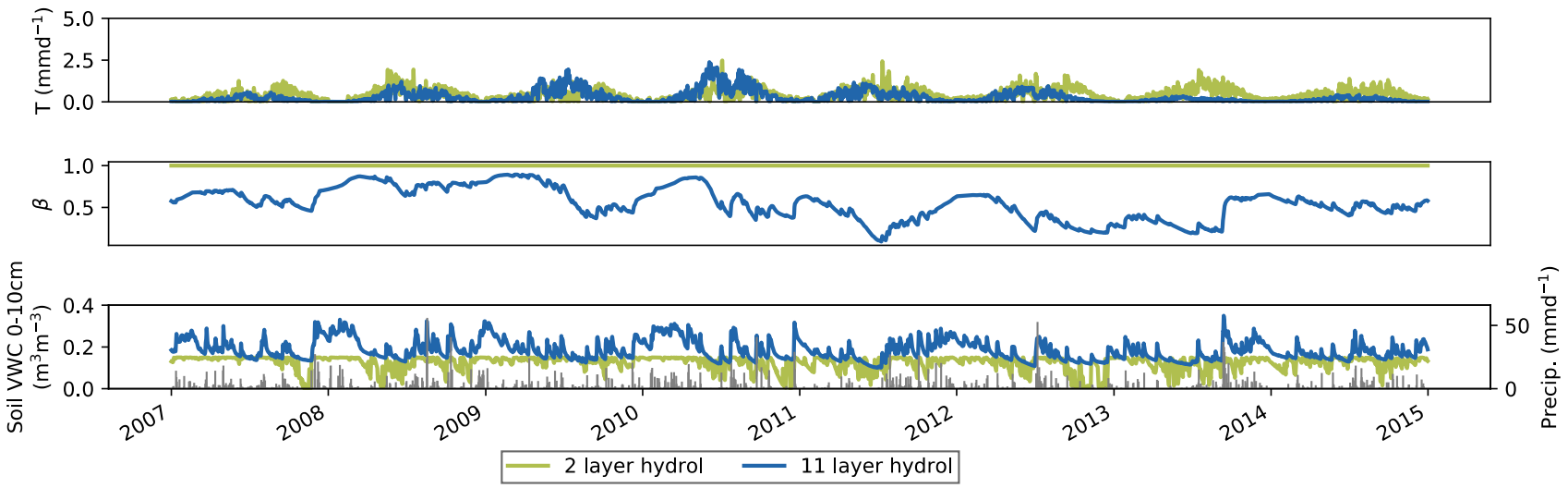
c) US-SRM

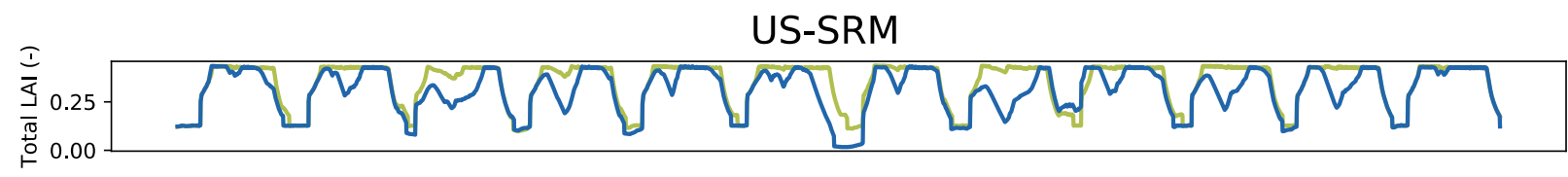

郎:

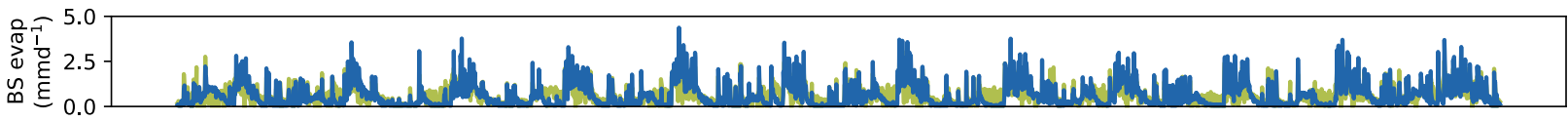

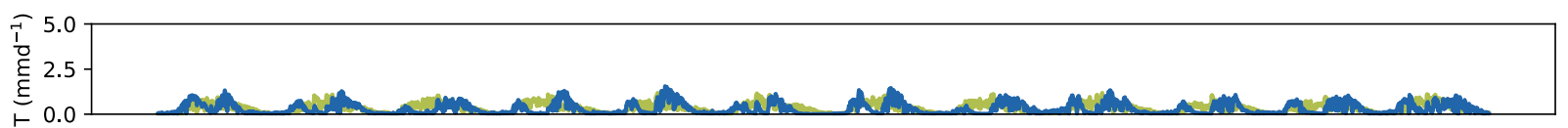

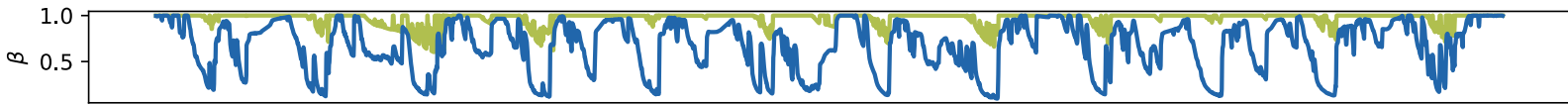

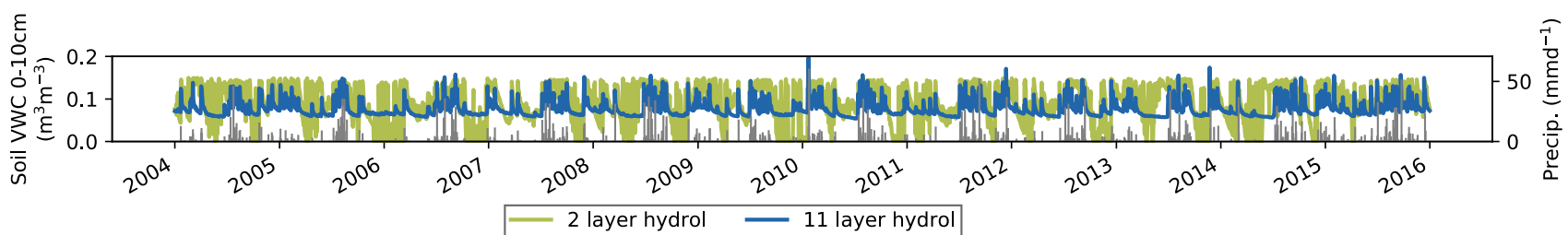


d) US-Whs

US-Whs

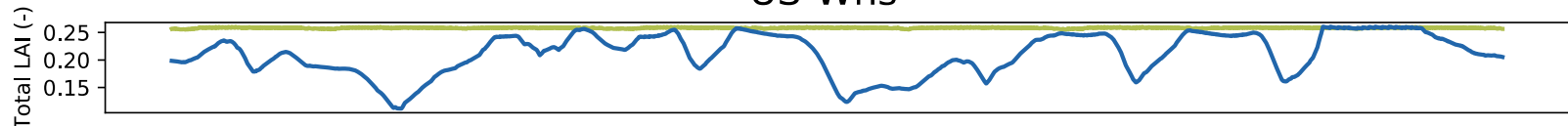

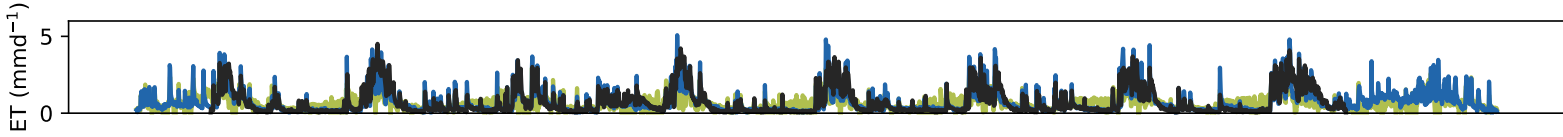

管

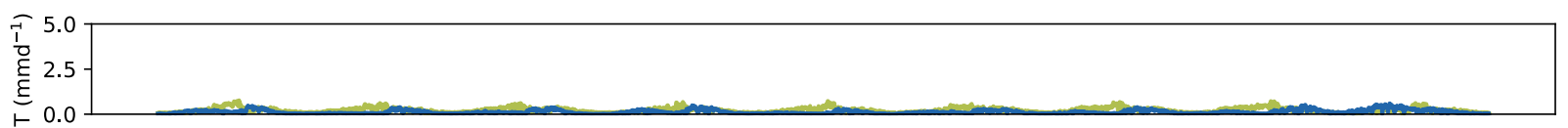

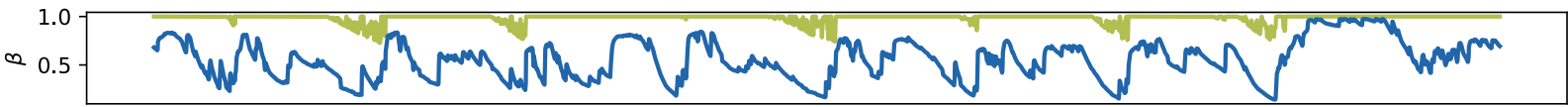

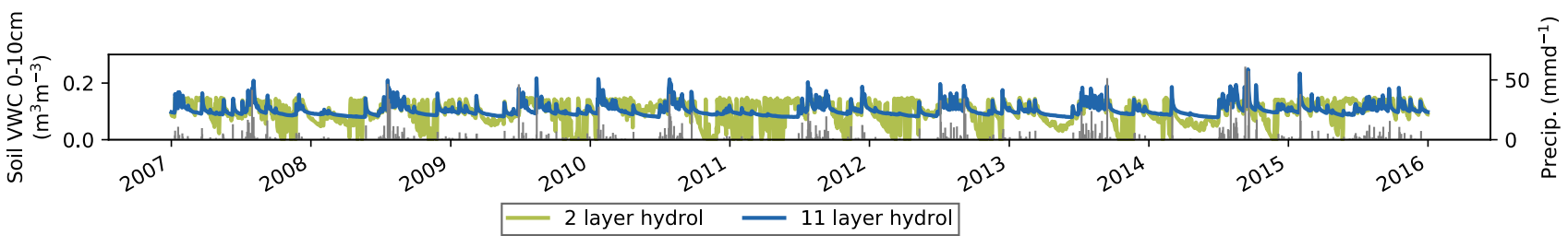


e) US-SRG
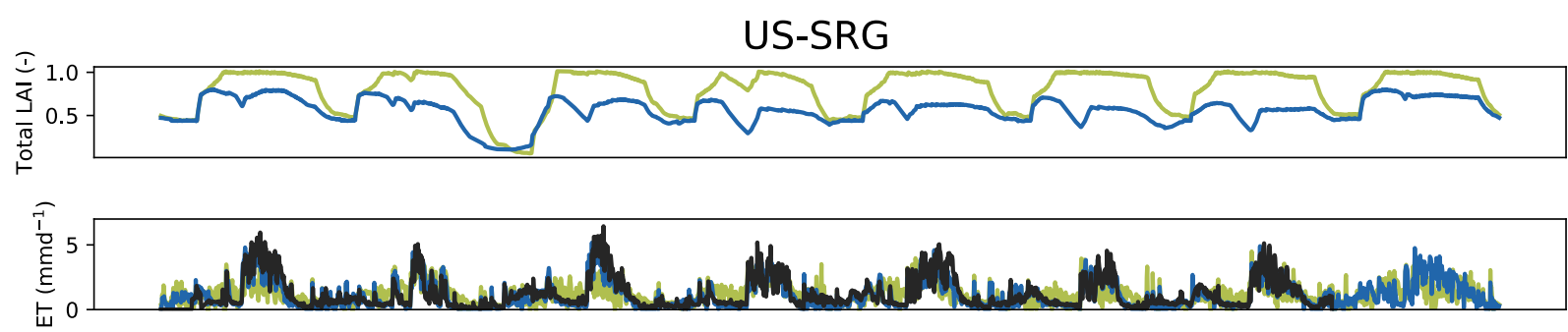

管

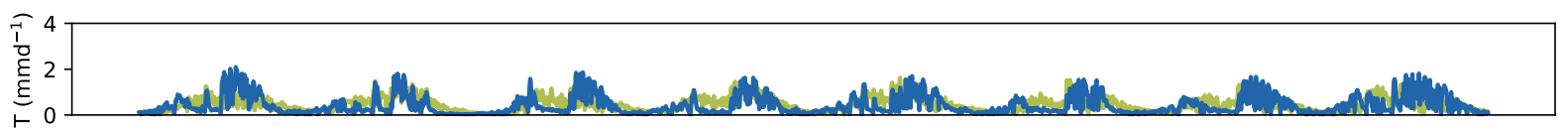

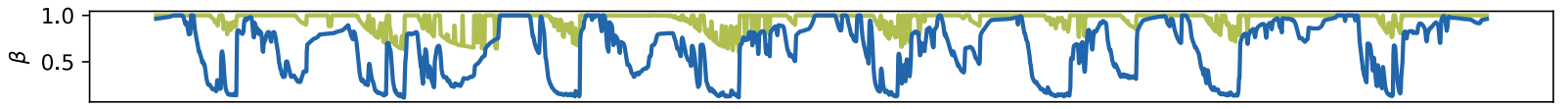

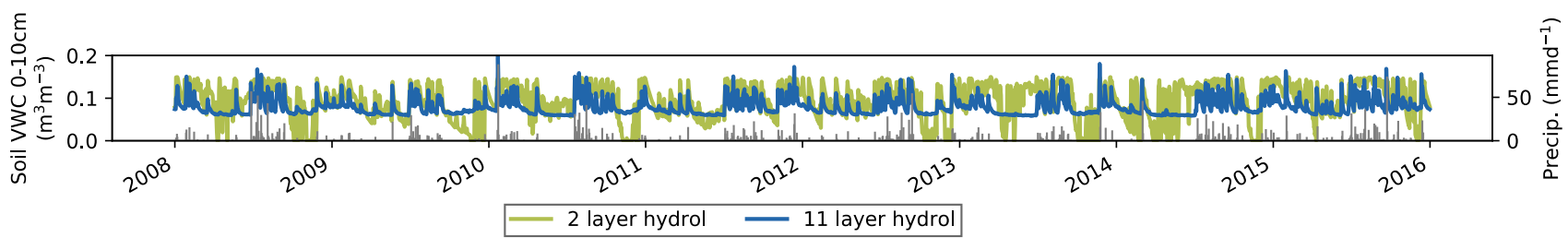




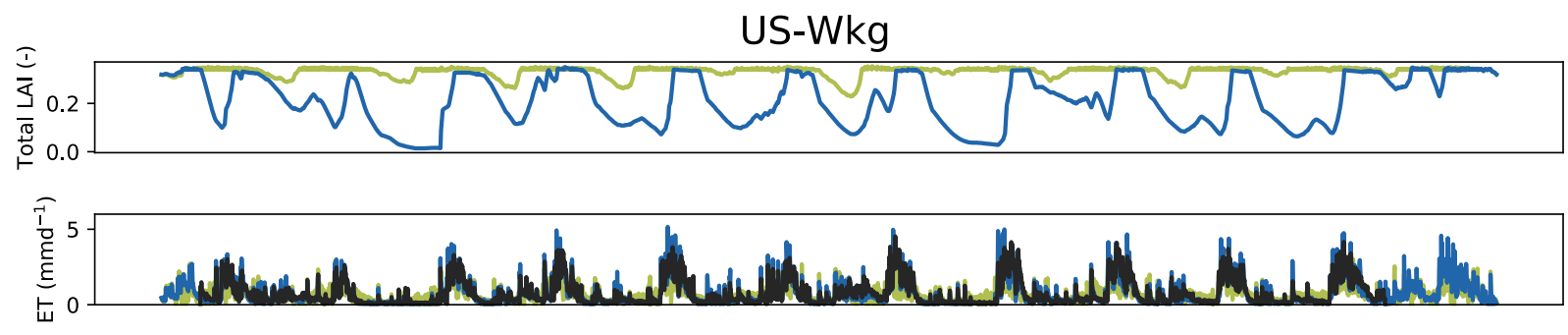

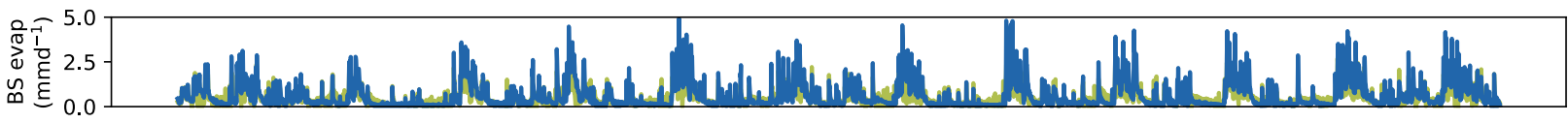

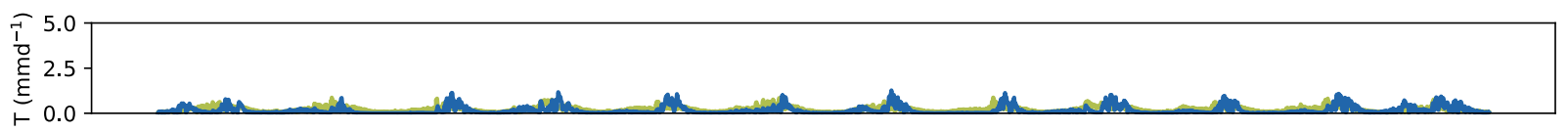

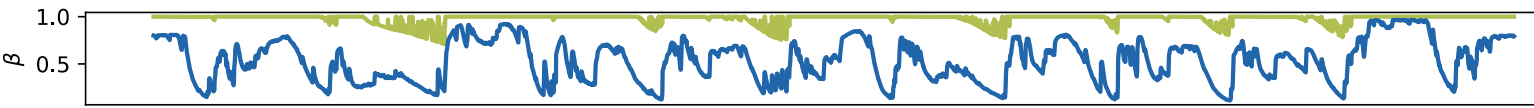

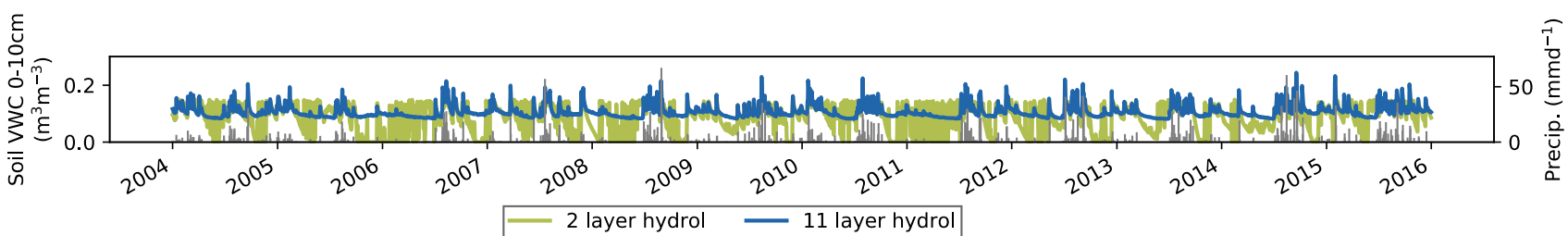


Figure S3: Monthly mean seasonal cycle for each site comparing the 2LAY (green curve) and 11LAY simulations (blue curve) with observations (black curve). Top left: ET; top right: T/ET ratios; bottom left: transpiration; bottom right: bare soil evaporation. Units in mmd-1. Sites in following order: a) US-Fuf; b) US-Vcp; c) US-SRM; d) US-Whs; e) US-SRG; f) US-Wkg.

a) US-Fuf
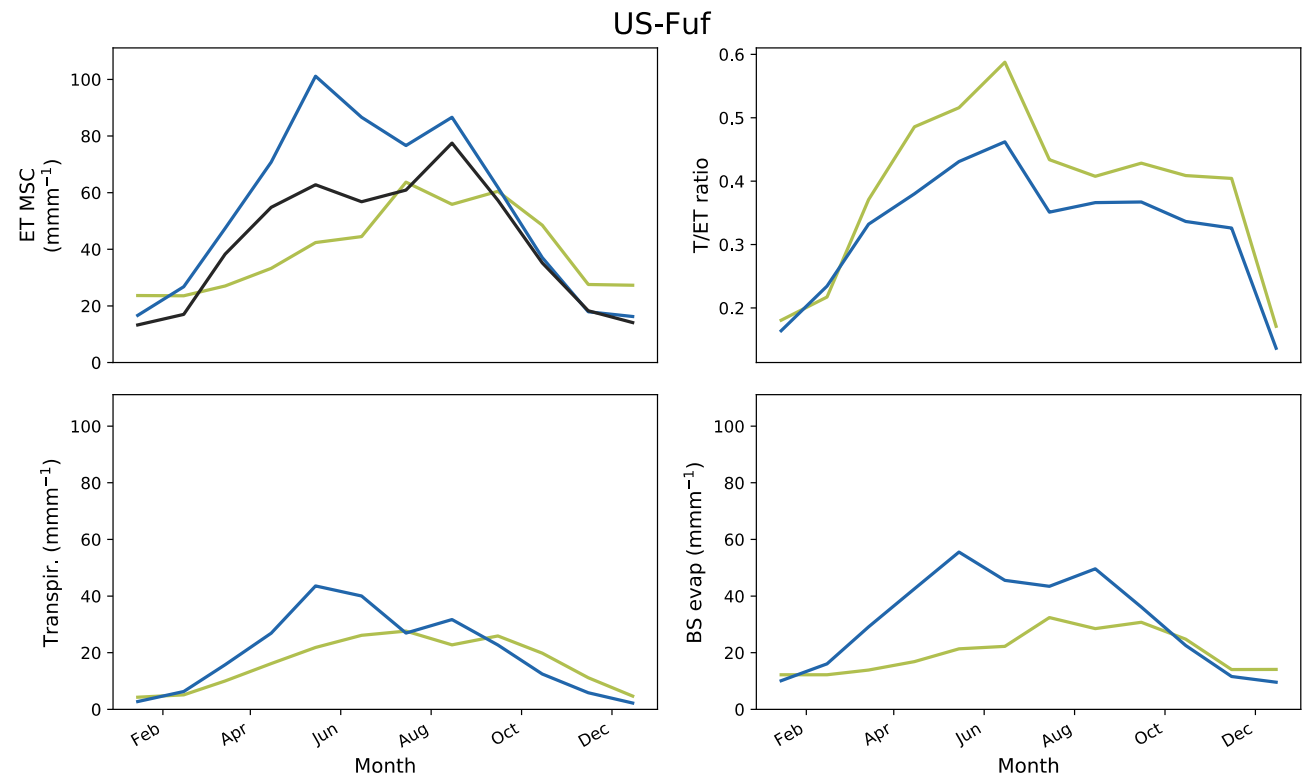

b) US-Vep
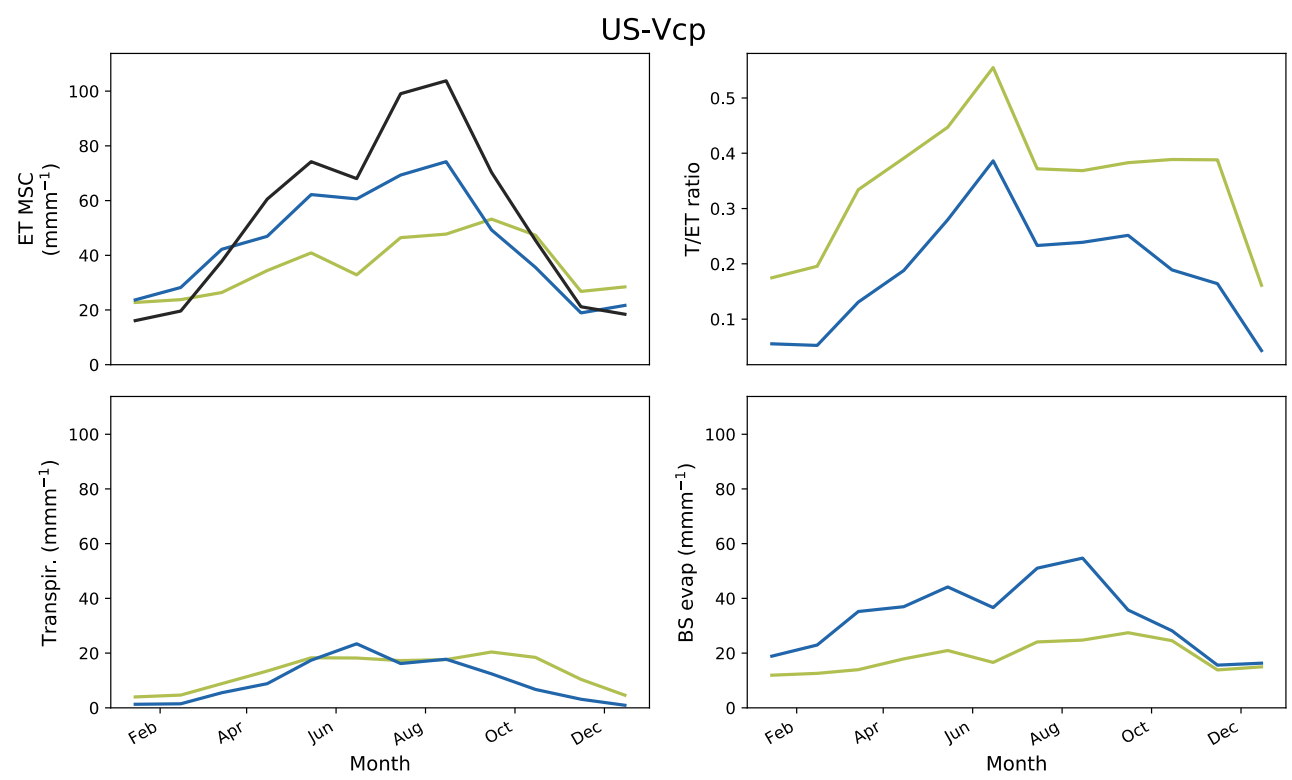
c) US-SRM
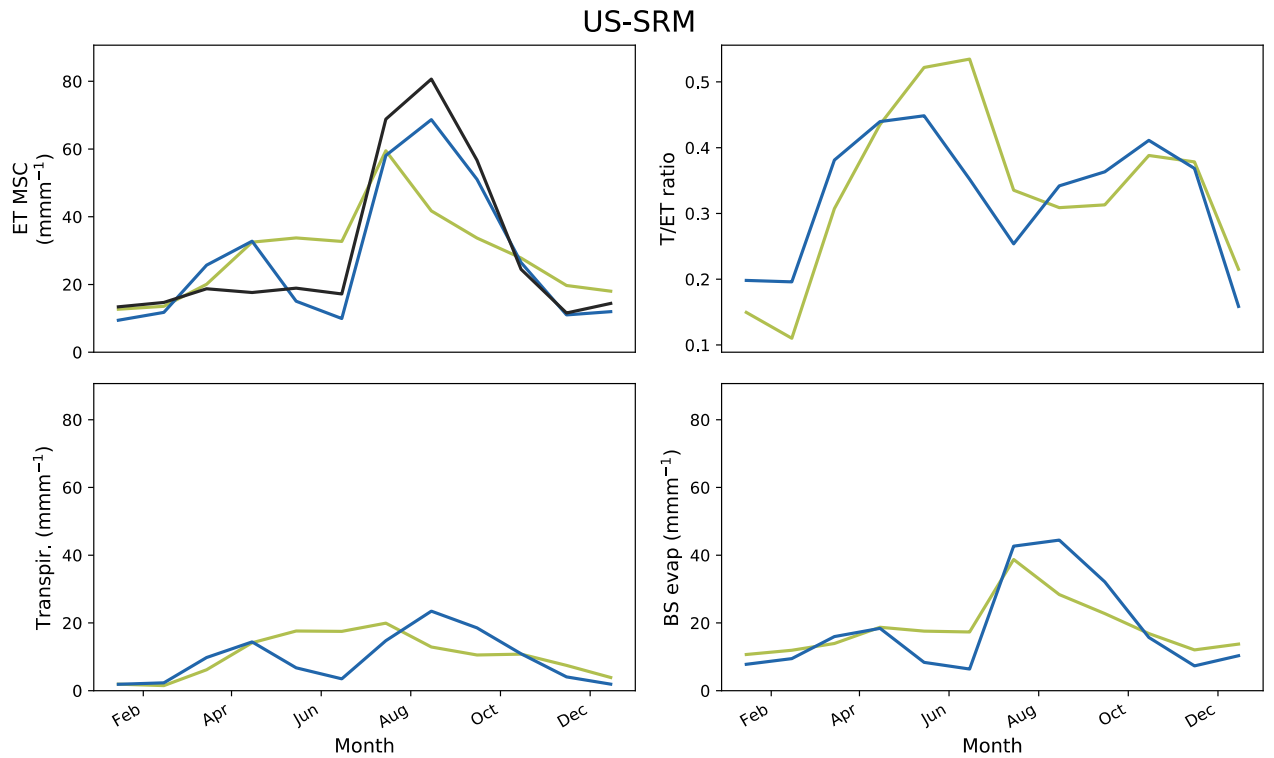

225

d) US-Whs
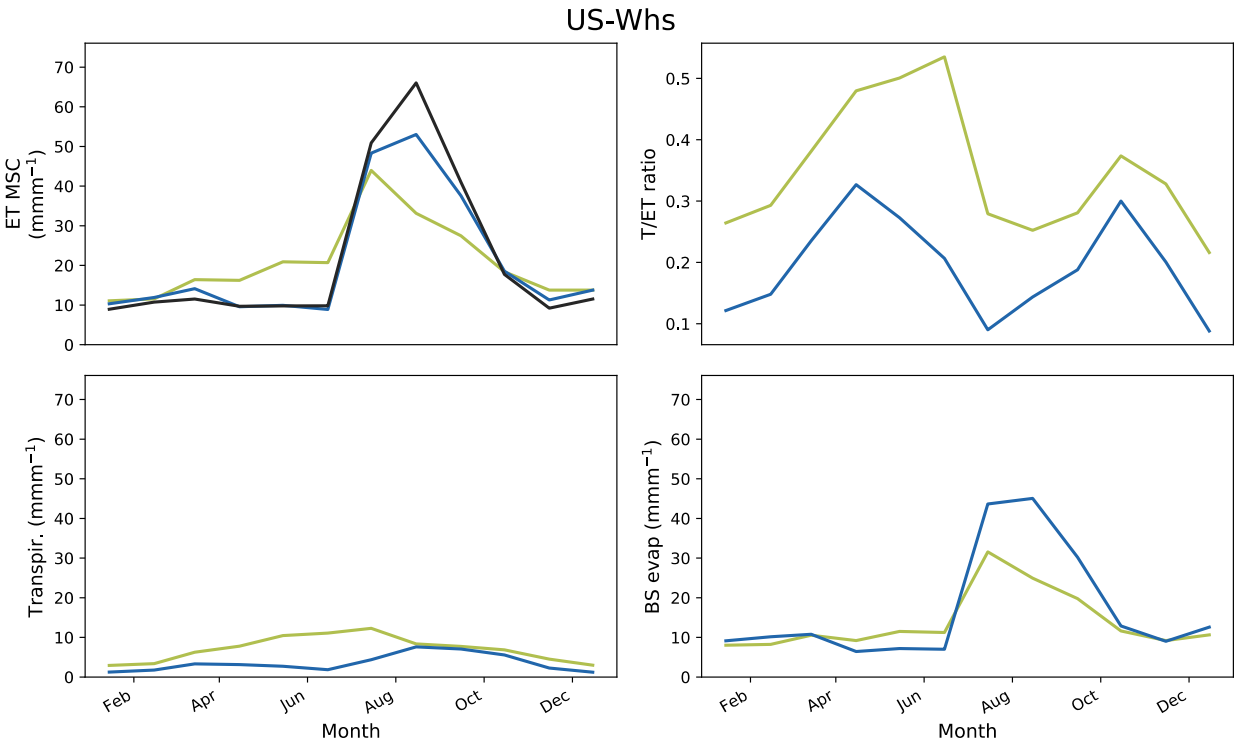

15 

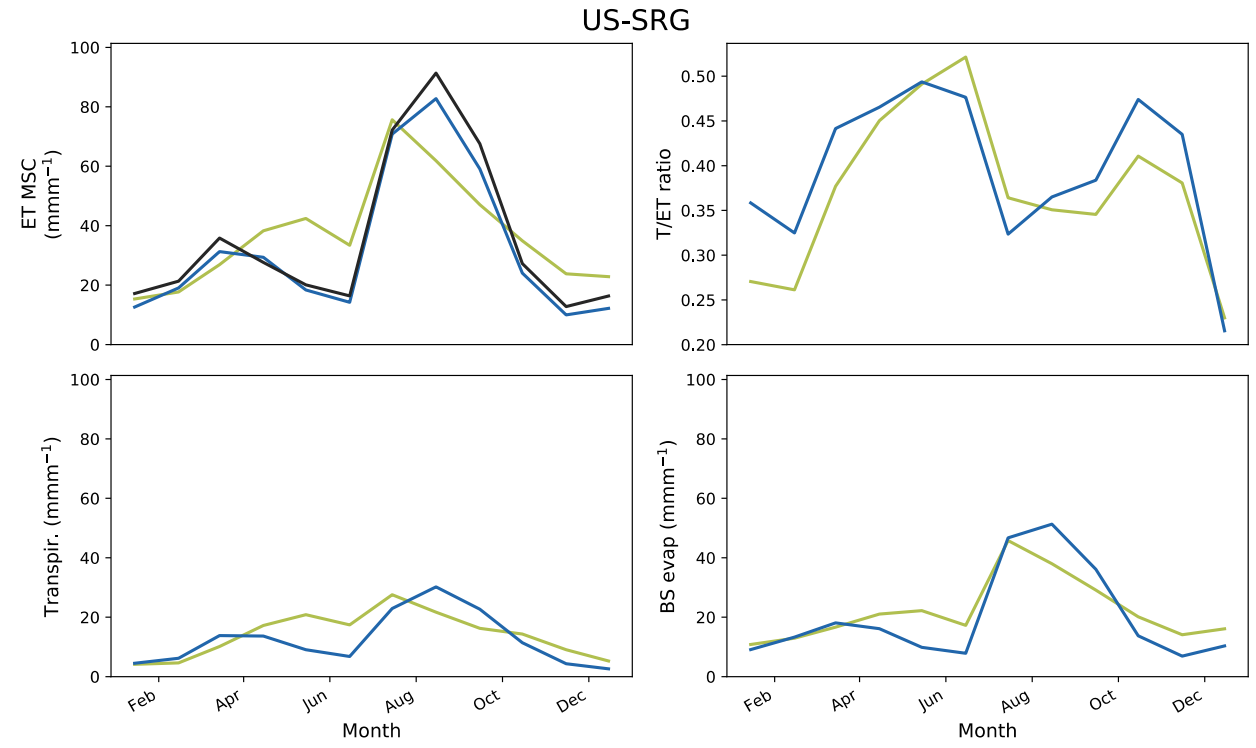

\section{f) US-W $\mathrm{kg}$}
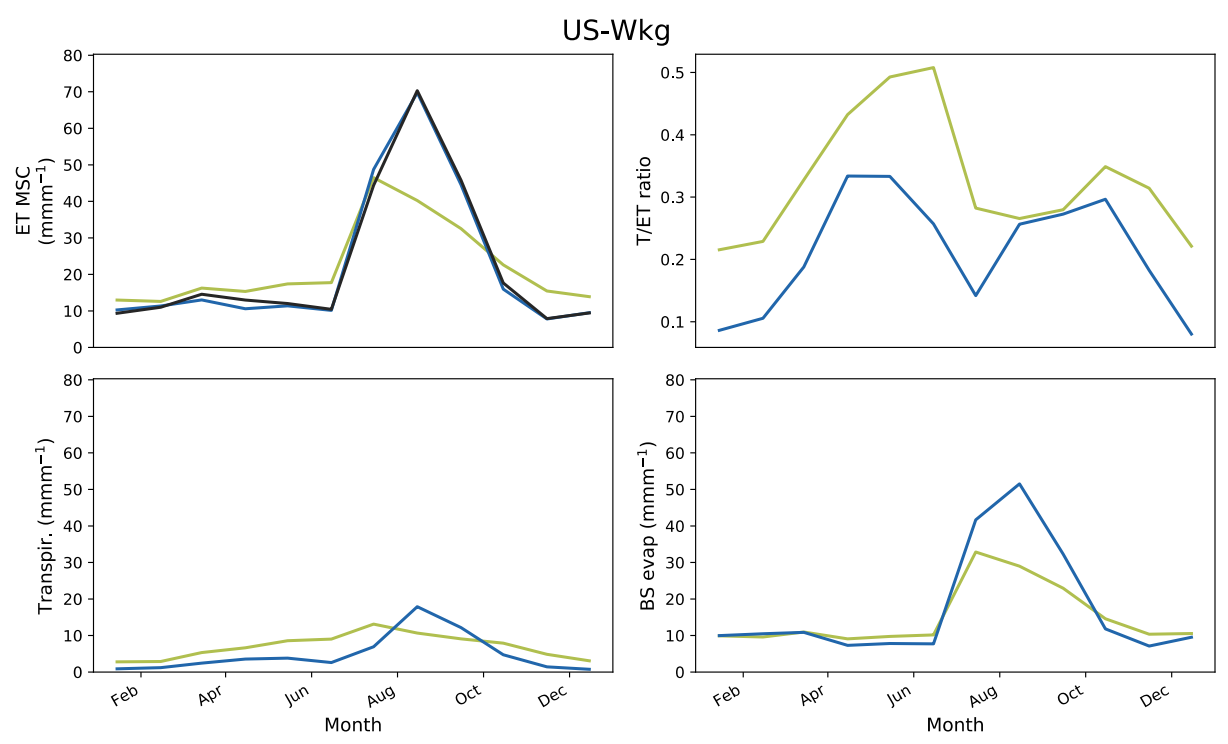
Figure S4: Daily simulated volumetric soil water content (VWC - m3m-3) across all site years compared to re-scaled (via linear CDF matching) observations at each site for three depths (upper, middle, lower) in the soil profile. The soil depths and their corresponding model layers are given in Table 2. Precipitation is shown in the grey lines in the bottom panel for each site.

a) US-Fuf
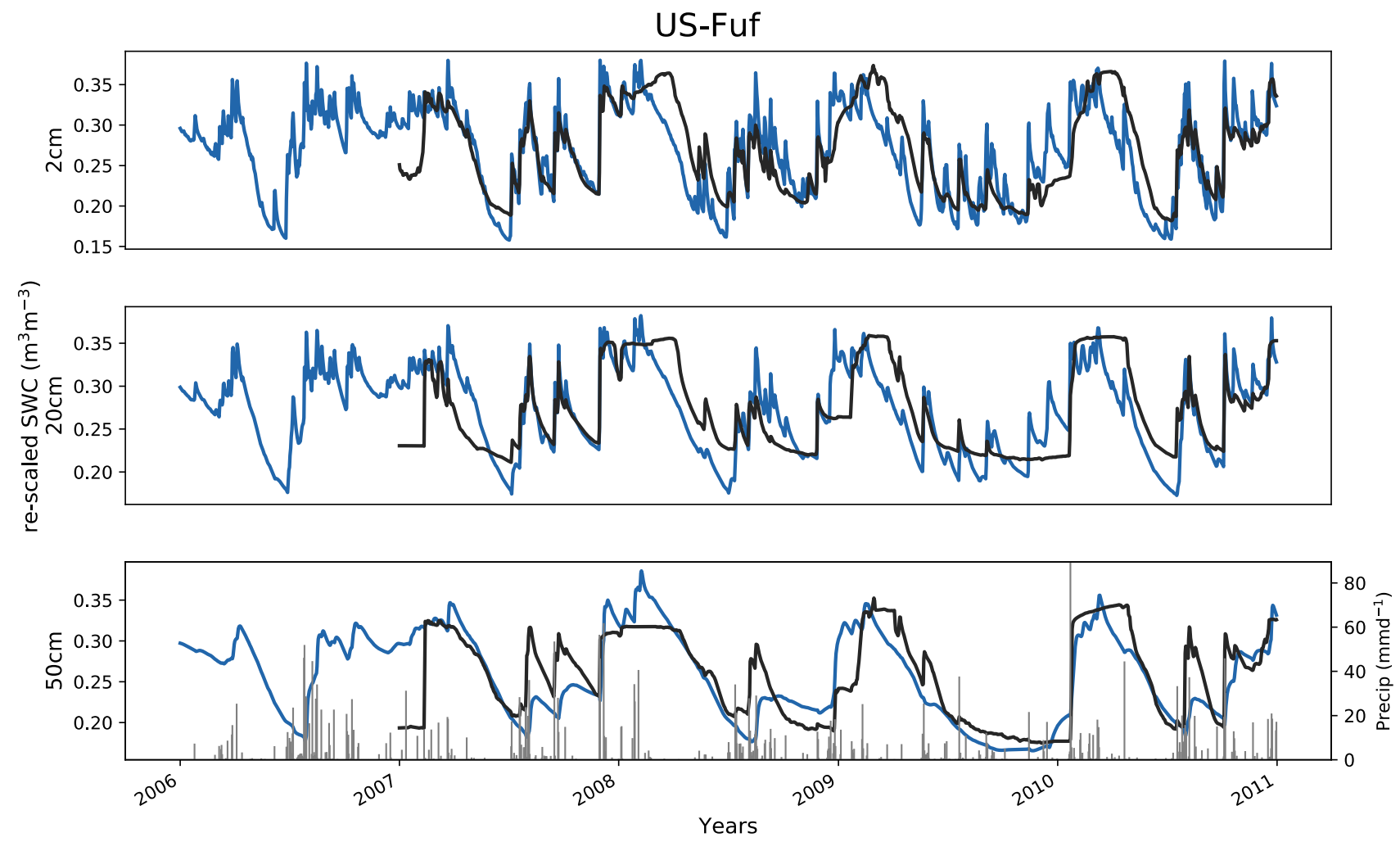
b) US-Vep

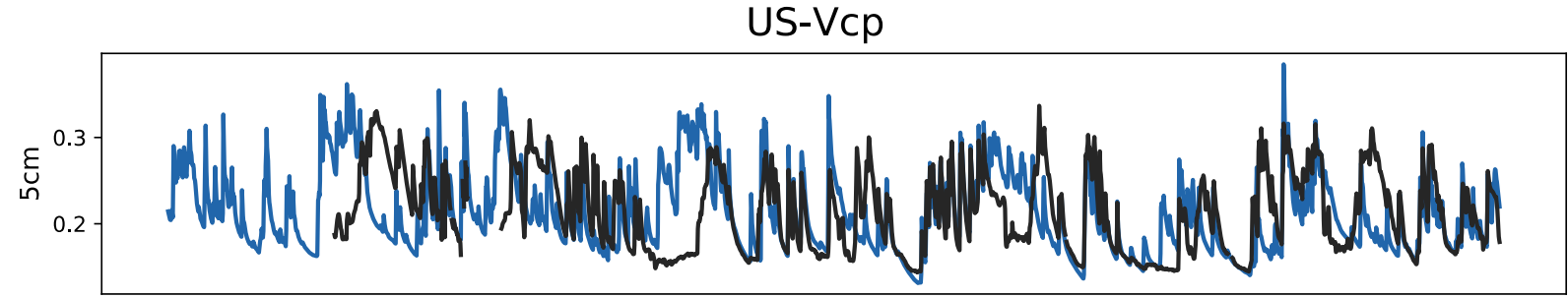

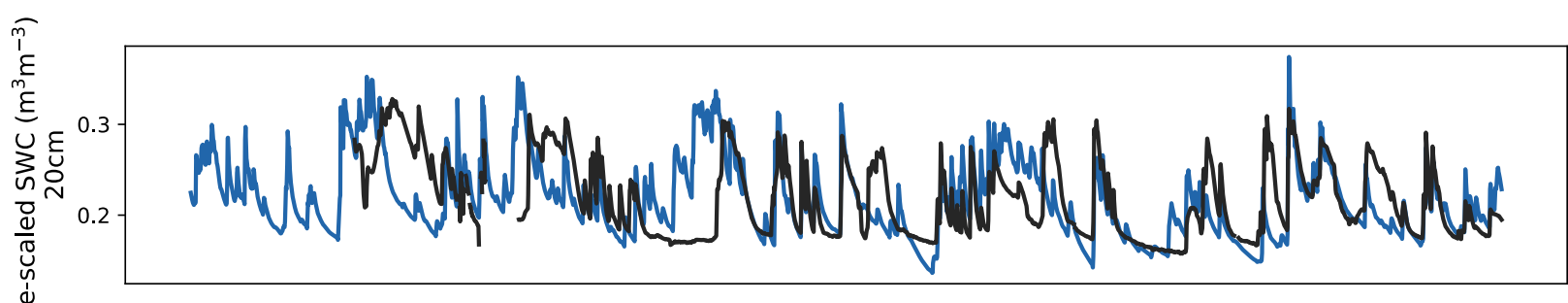

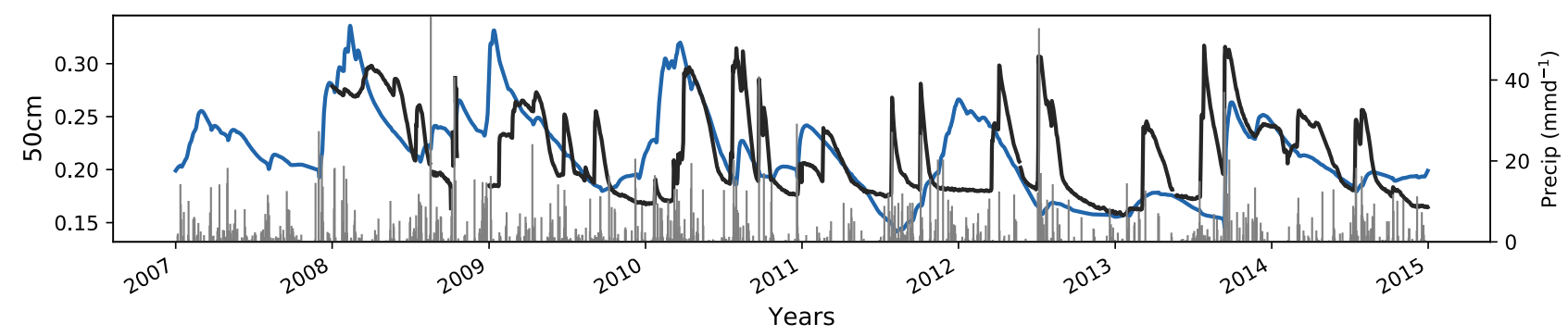


c) US-SRM
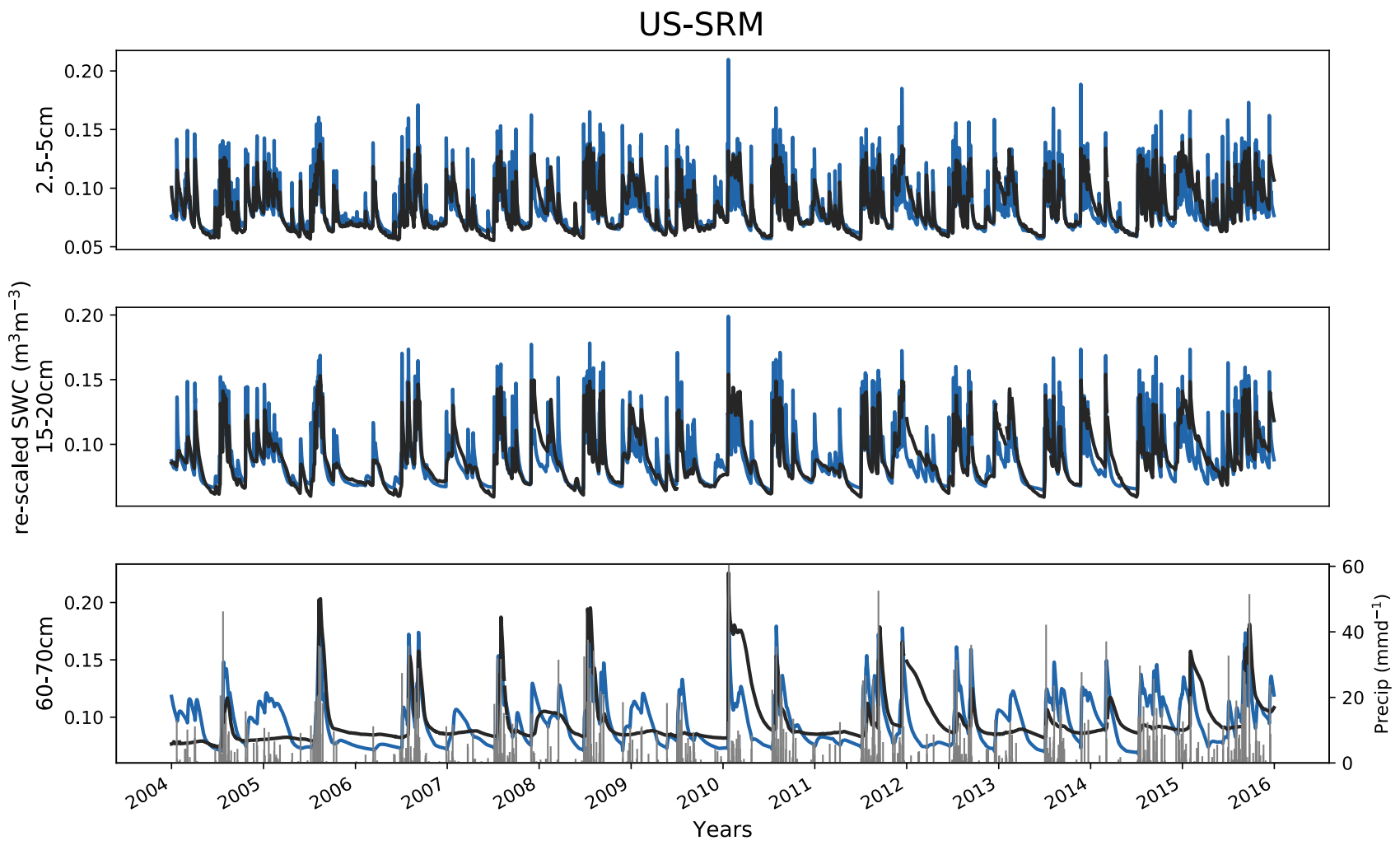
d) US-Whs

US-Whs
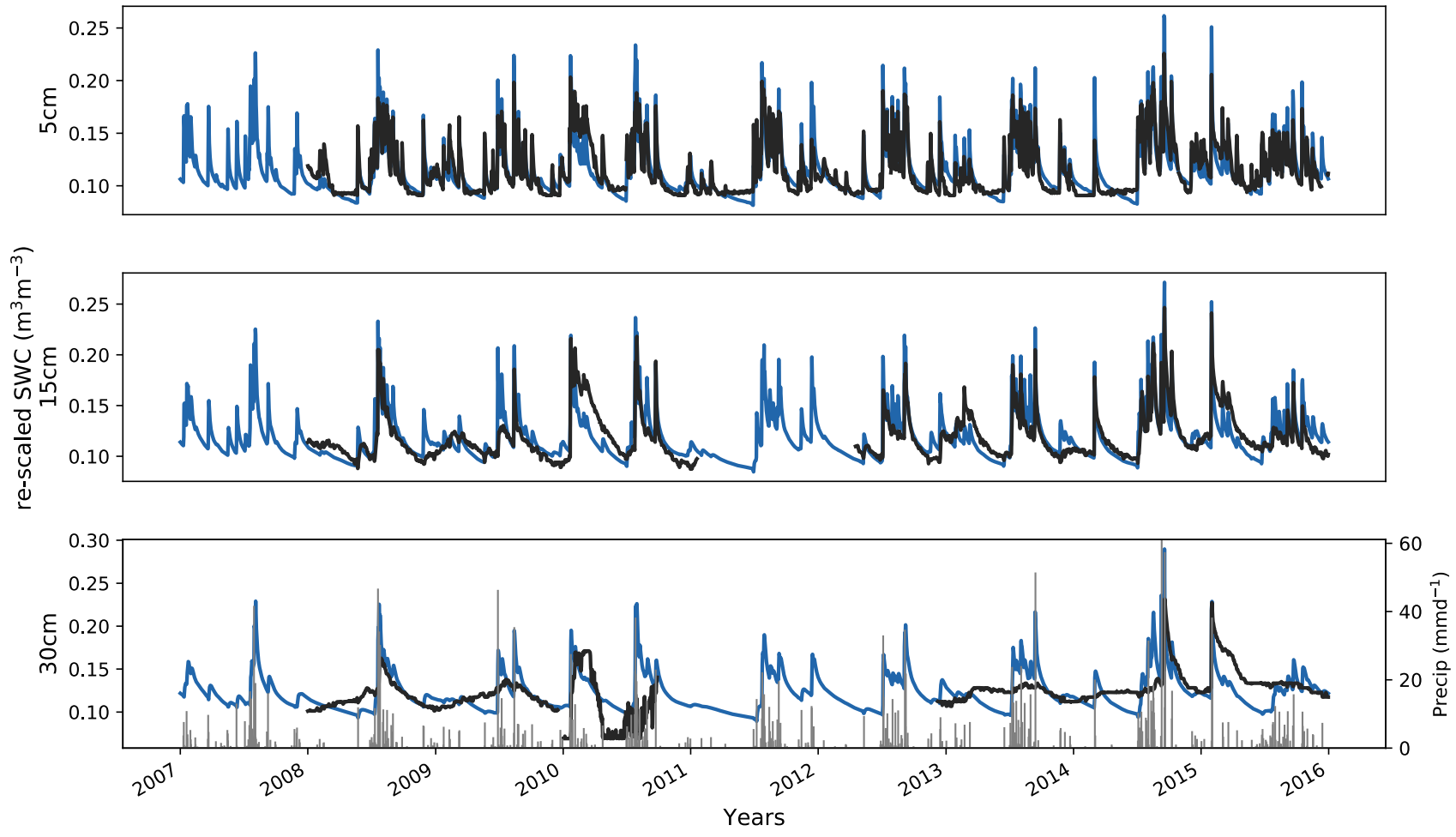
e) US-SRG

US-SRG

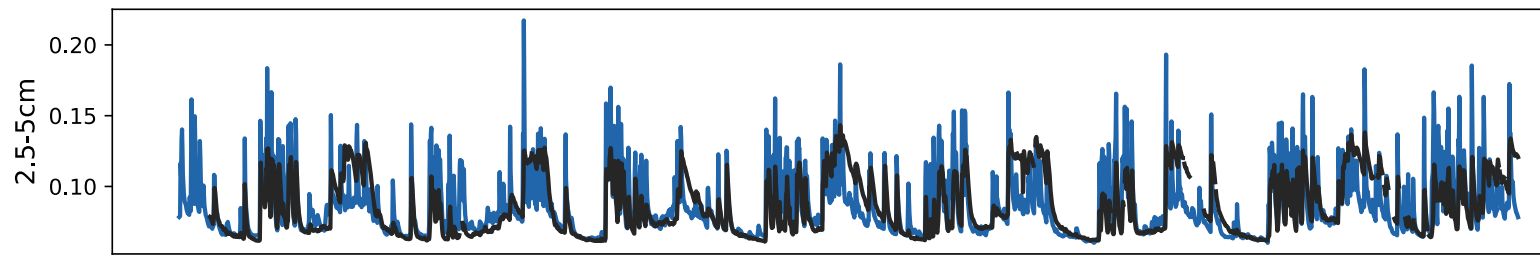

Find

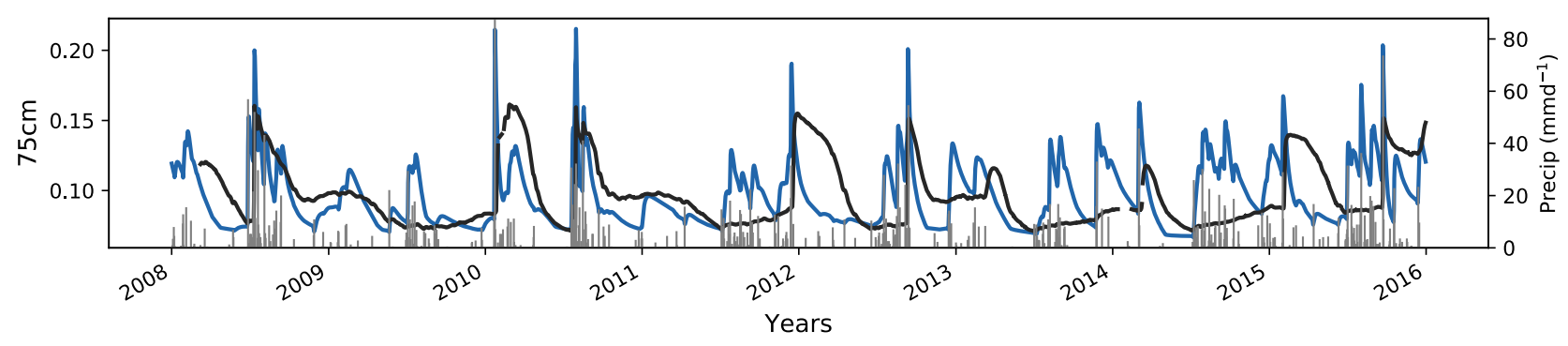

305 
f) US-Wkg
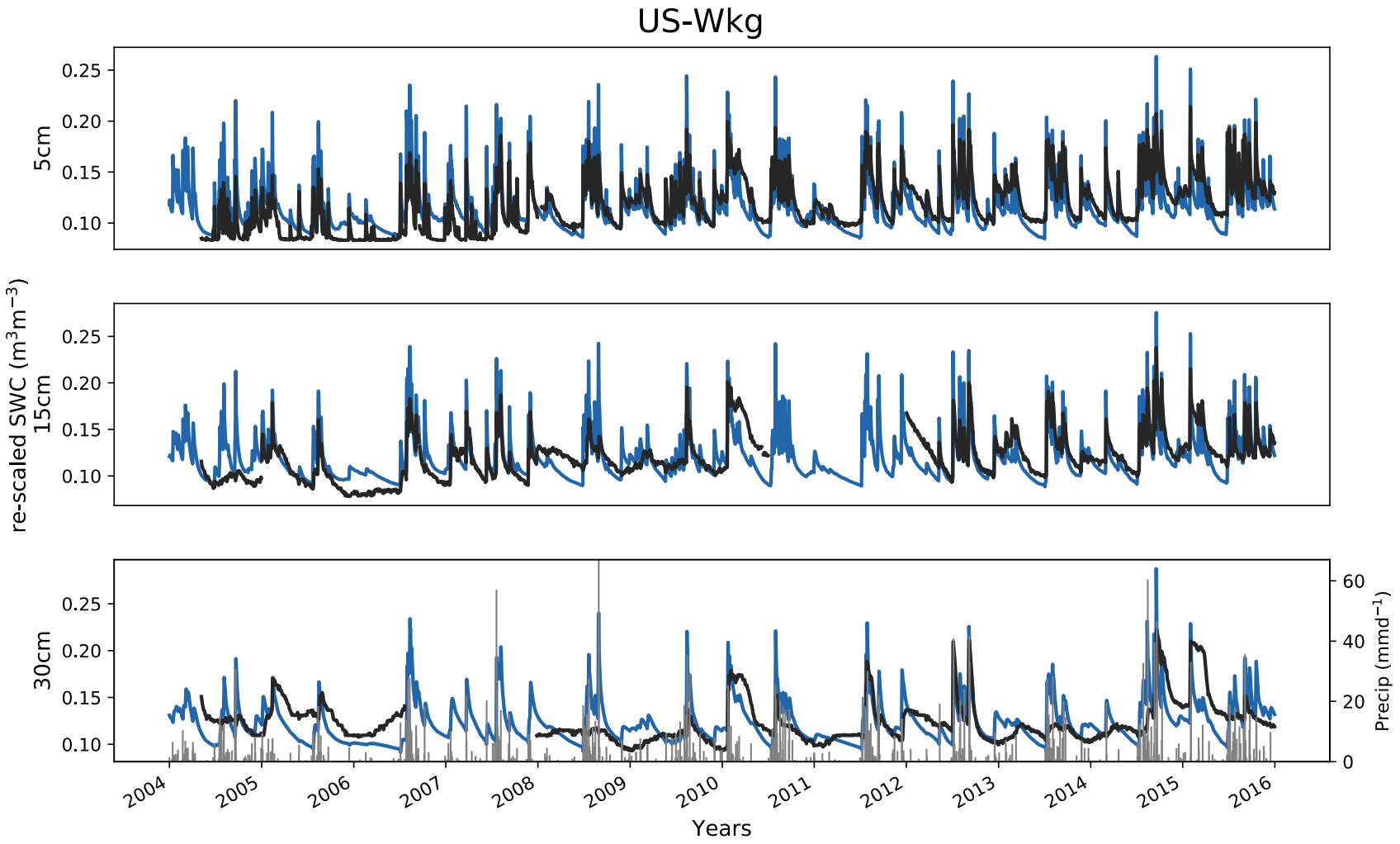
Figure S5: Linear regressions between spring (March-April) mean monthly LAI and spring mean ET model-data misfits for each site. The dominant PFT is given in brackets for each site. See Table 1 for PFT acronyms.
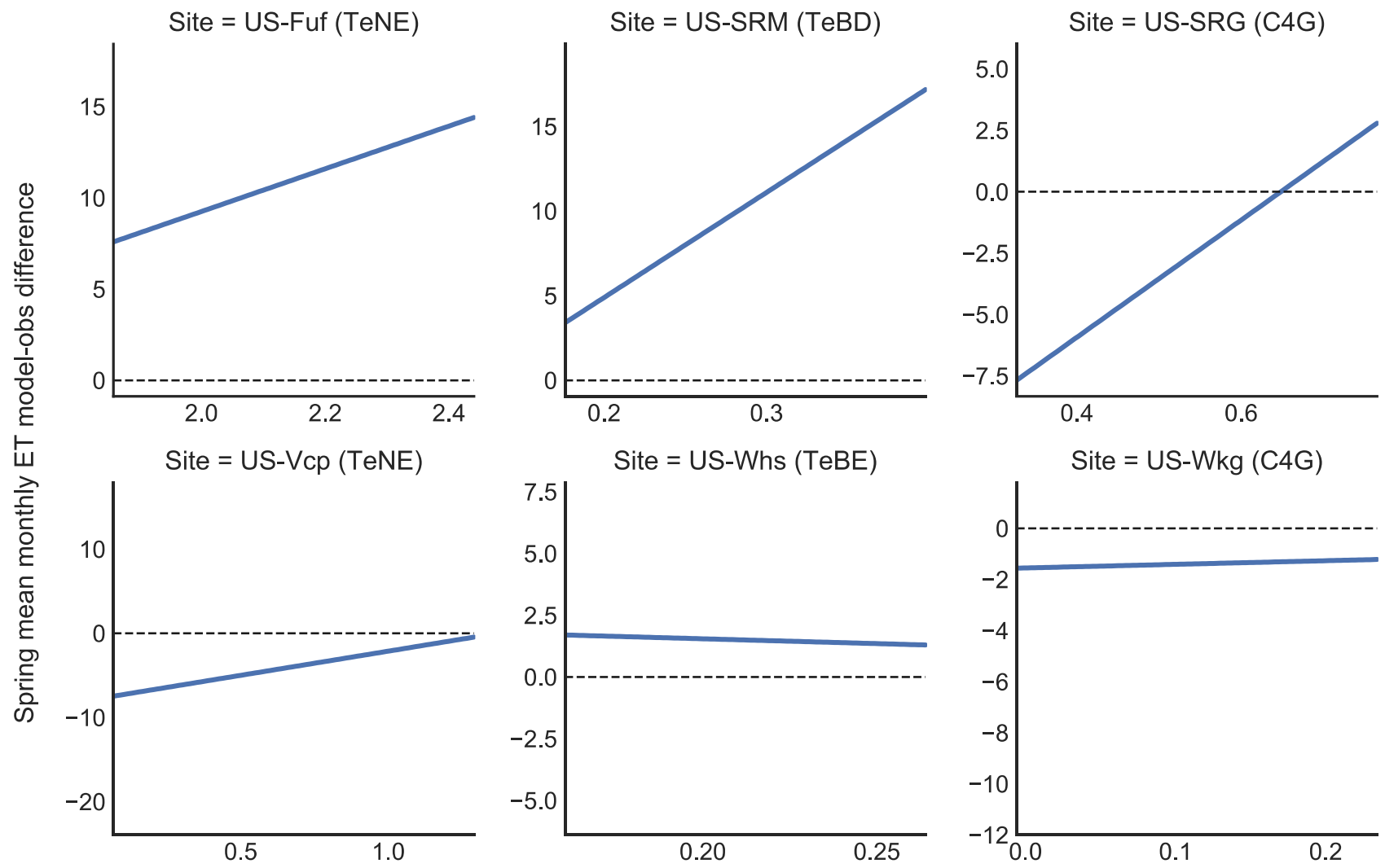

Spring (March-April) mean monthly LAI 
Figure S6: Plots comparing ET and LAI for C4 grasses (C4G) and mesquite shrubs (Temperate Broadleaved Deciduous - TeBD PFT in ORCHIDEE) monthly mean seasonal cycles at US-SRM for the 2LAY (green curve) and 11LAY (blue curve) model versions in comparison to observations (black curve).
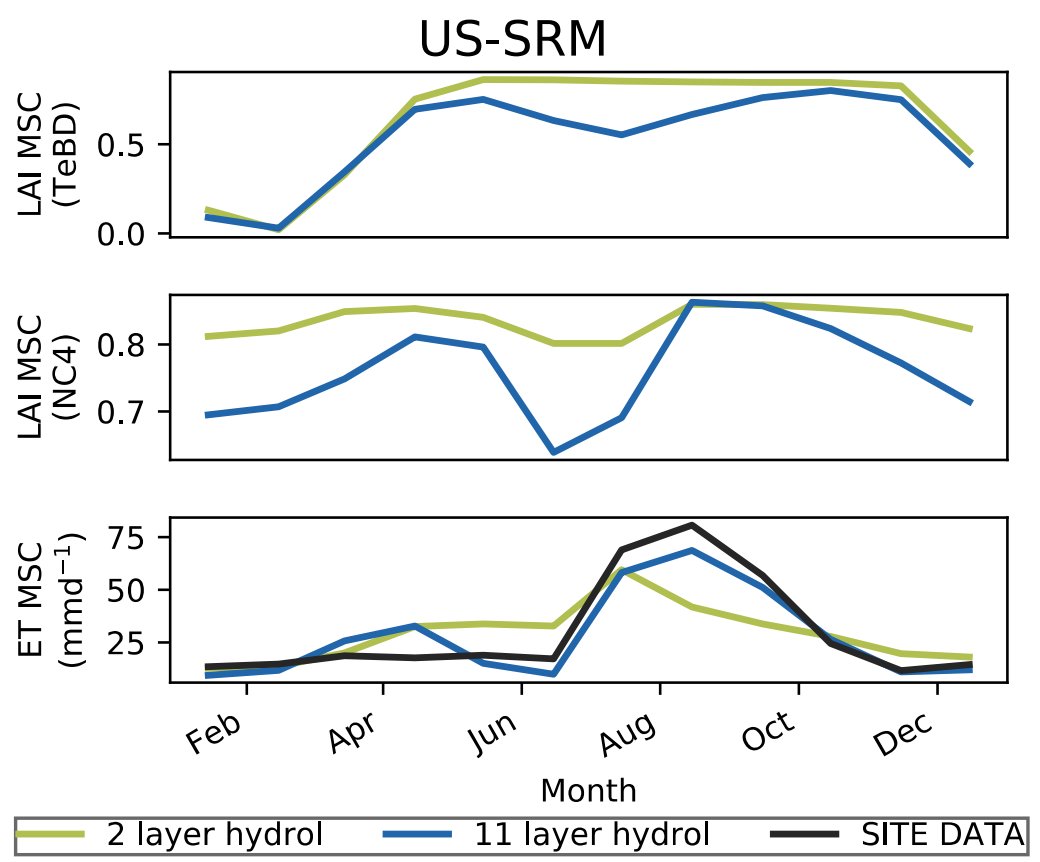
Figure S7: Linear regressions between monsoon (July-September) mean monthly LAI and monsoon mean ET model-data misfits for each site. The dominant PFT is given in brackets for each site. See Table 1 for PFT acronyms.
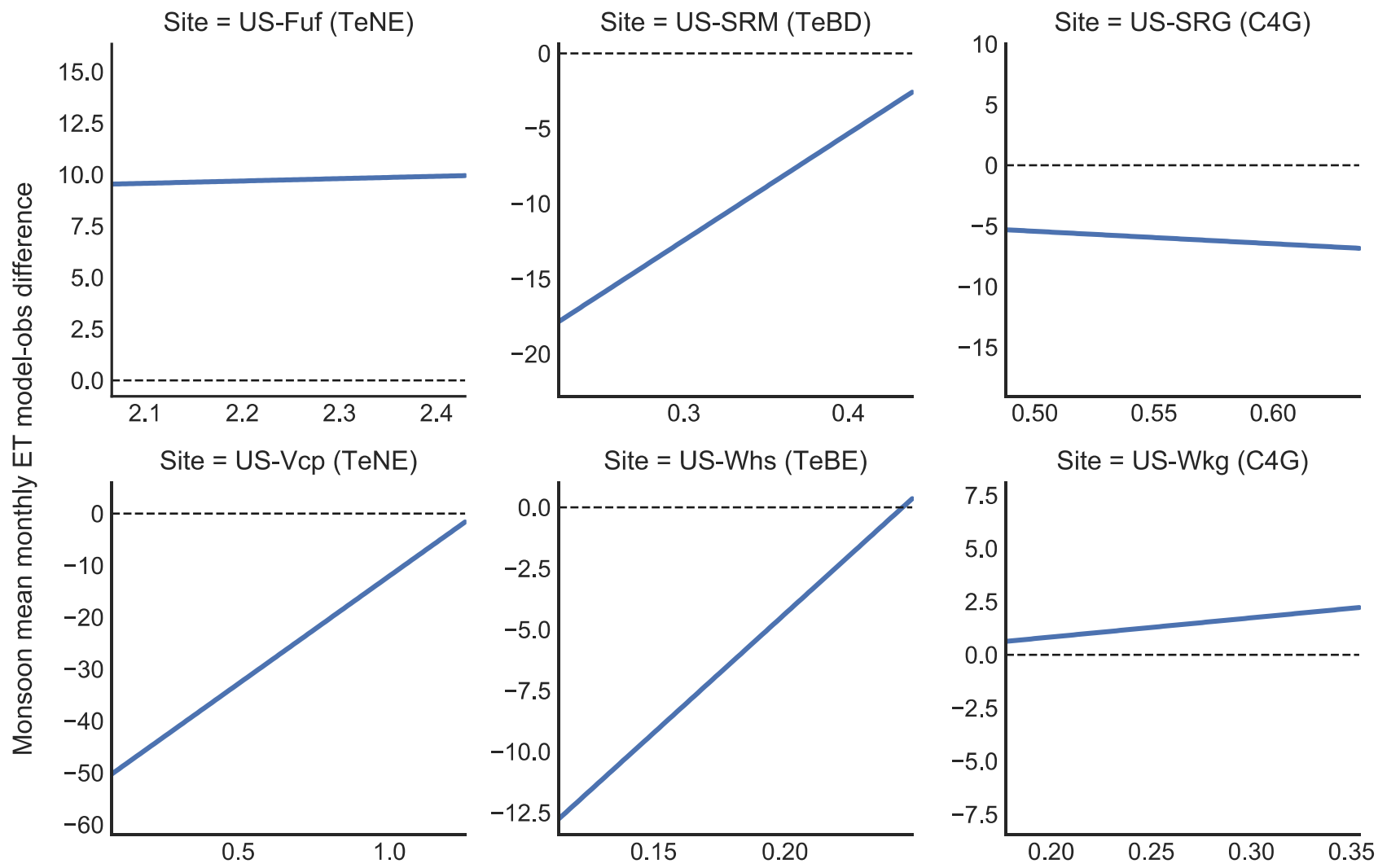

Monsoon (Jul-Sept) mean monthly LAI 
Figure S8: ET monthly mean seasonal cycle for all low elevation sites comparing the default 11LAY simulations (blue curve) with a simulation that increased the $\mathrm{C} 4$ grass fraction at the expense of the bare soil fraction (yellow curve). ET is compared to observations (black curve). Units in mmmonth-1.
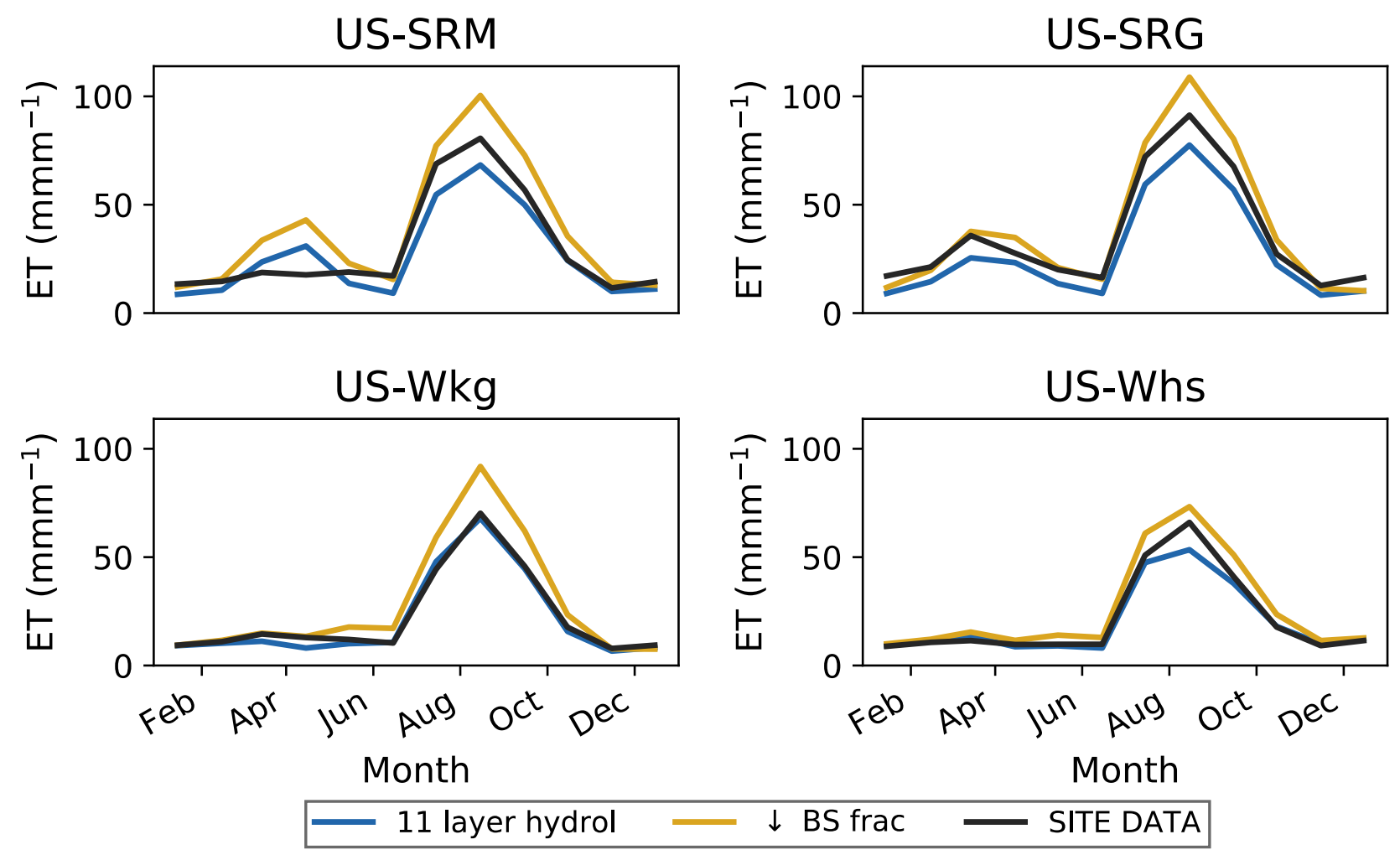
Figure S9: Monthly mean seasonal cycle for all sites comparing the default 11LAY simulations (blue curve) with a simulation that included an additional bare soil evaporation resistance term (red curve). ET is compared to observations (black curve). In all subfigures - top right: T/ET ratios; bottom left: transpiration; bottom right: bare soil evaporation. Units in mmmonth-1.

a) US-Fuf
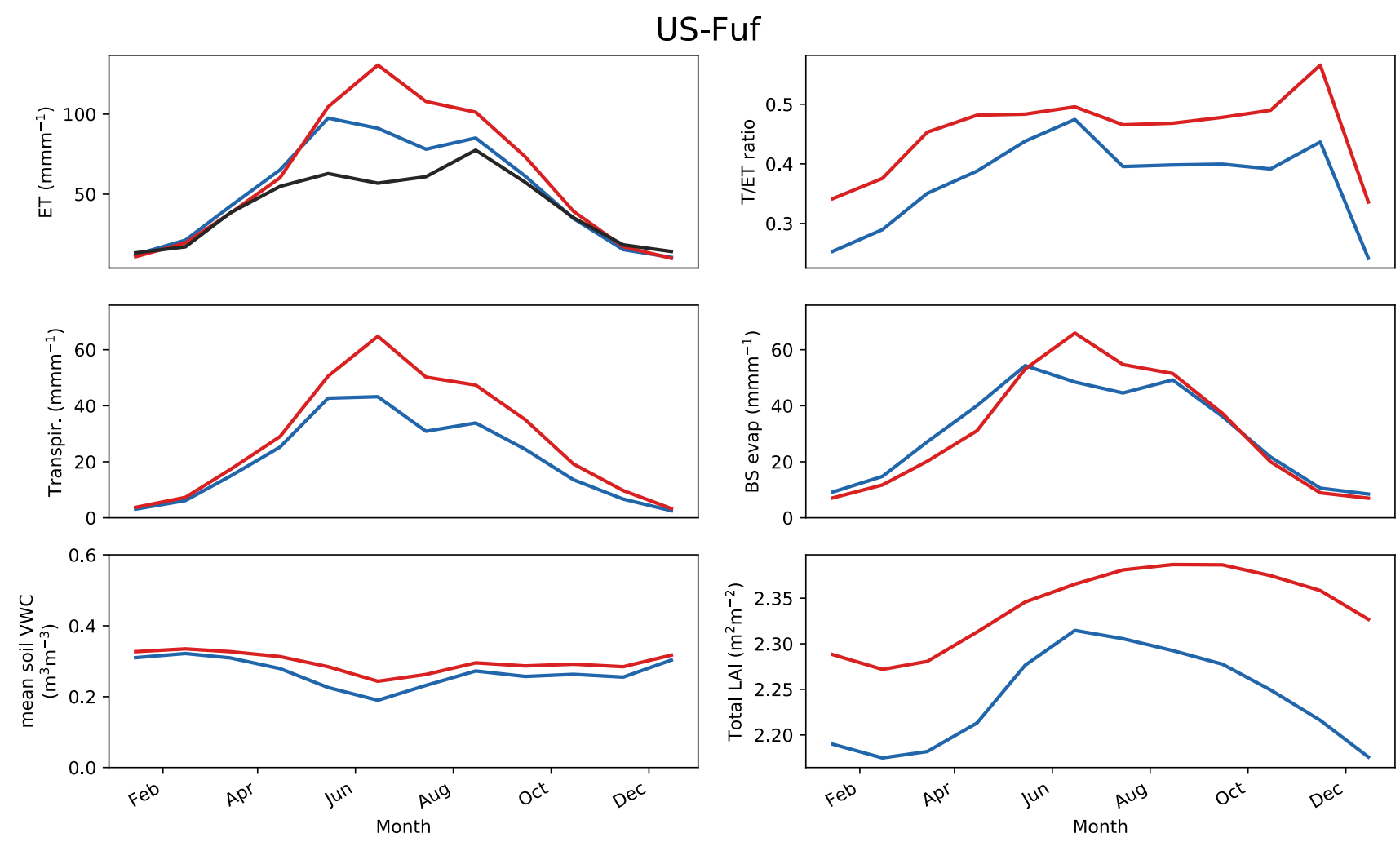

- 11 layer hydrol $\longrightarrow$ Bare soil evap resistance $\quad$ SITE DATA 
b) US-Vcp
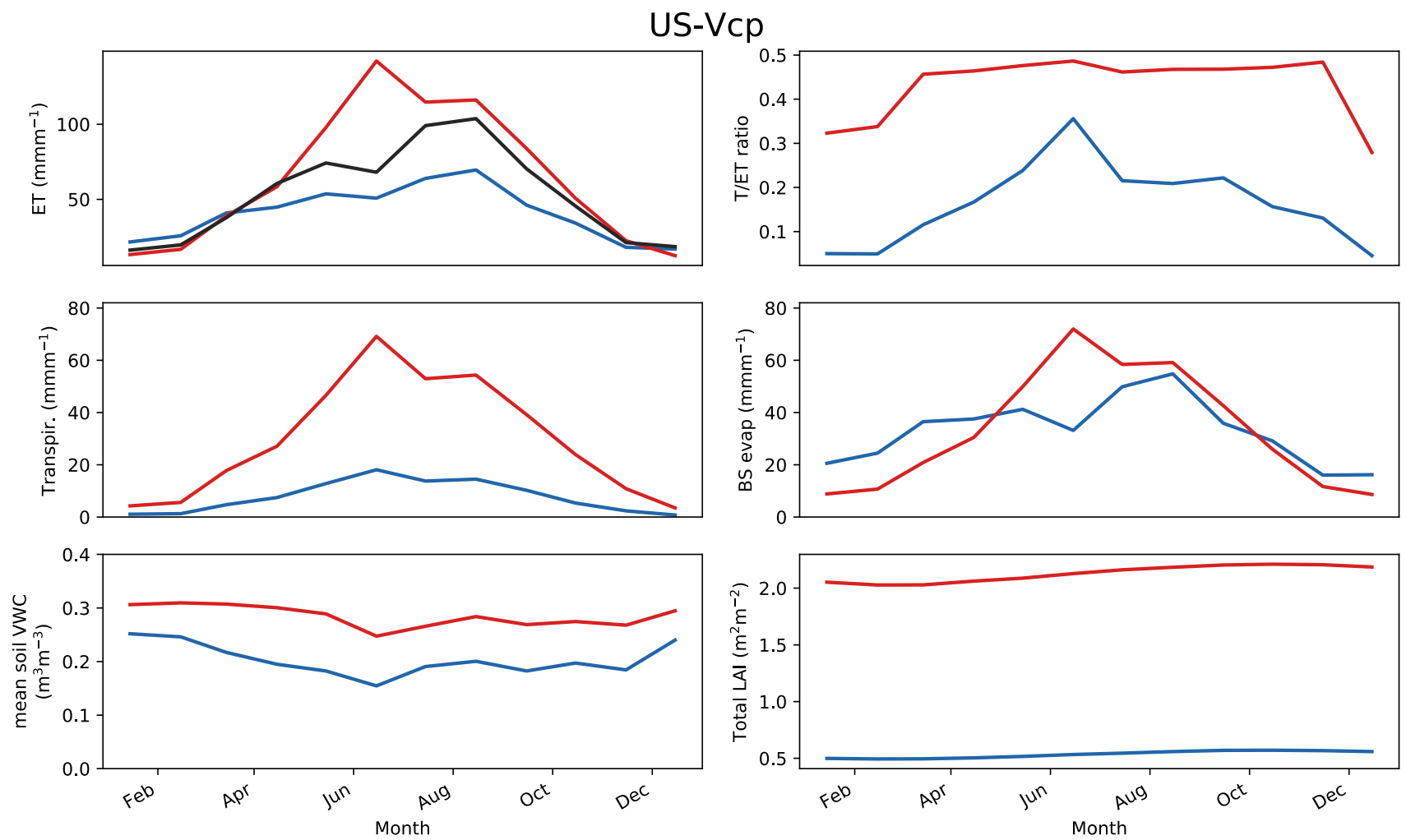

420

— 11 layer hydrol $\quad$ Bare soil evap resistance - SITE DATA 

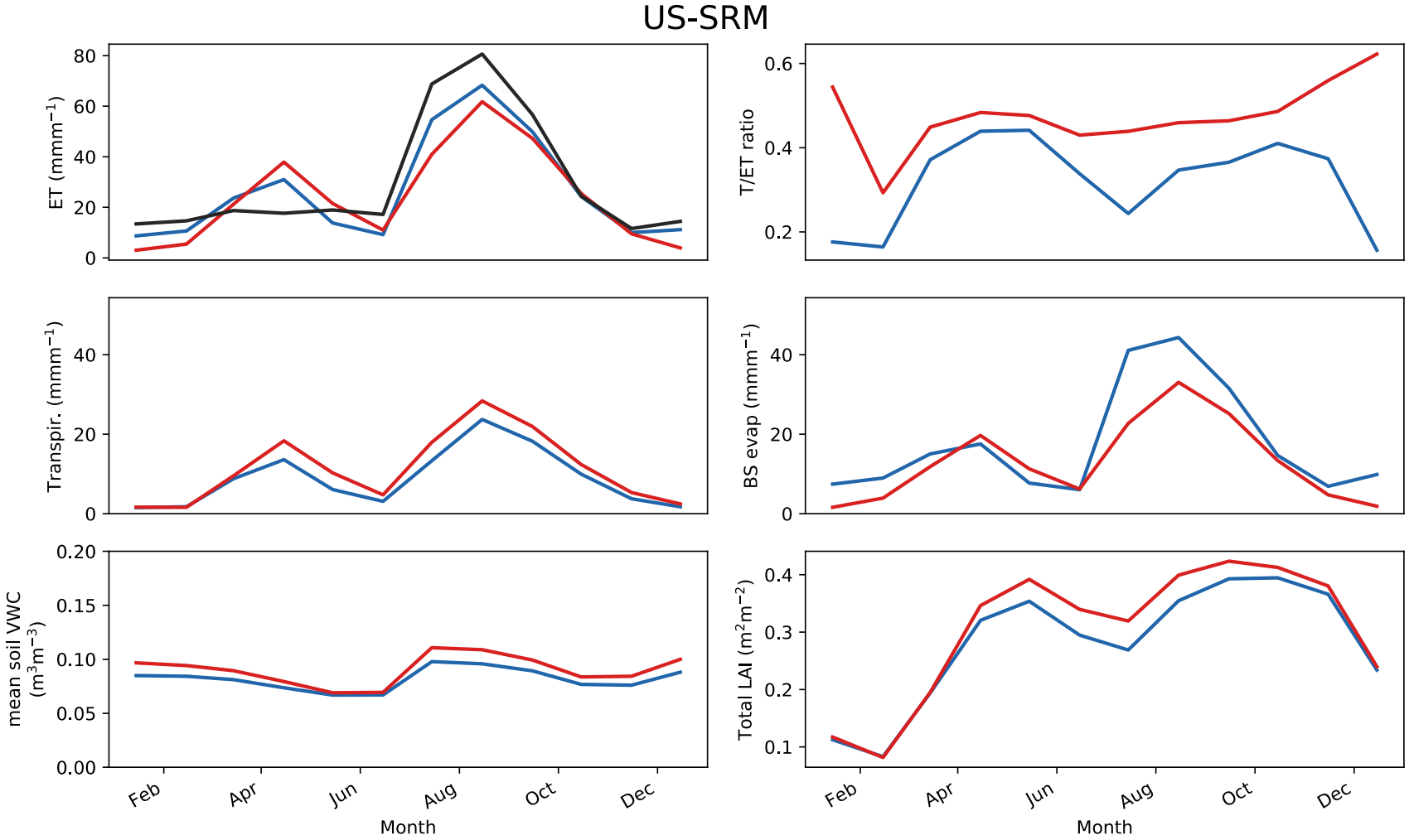

11 layer hydrol - Bare soil evap resistance - SITE DATA 


\section{d) US-Whs}

\section{US-Whs}
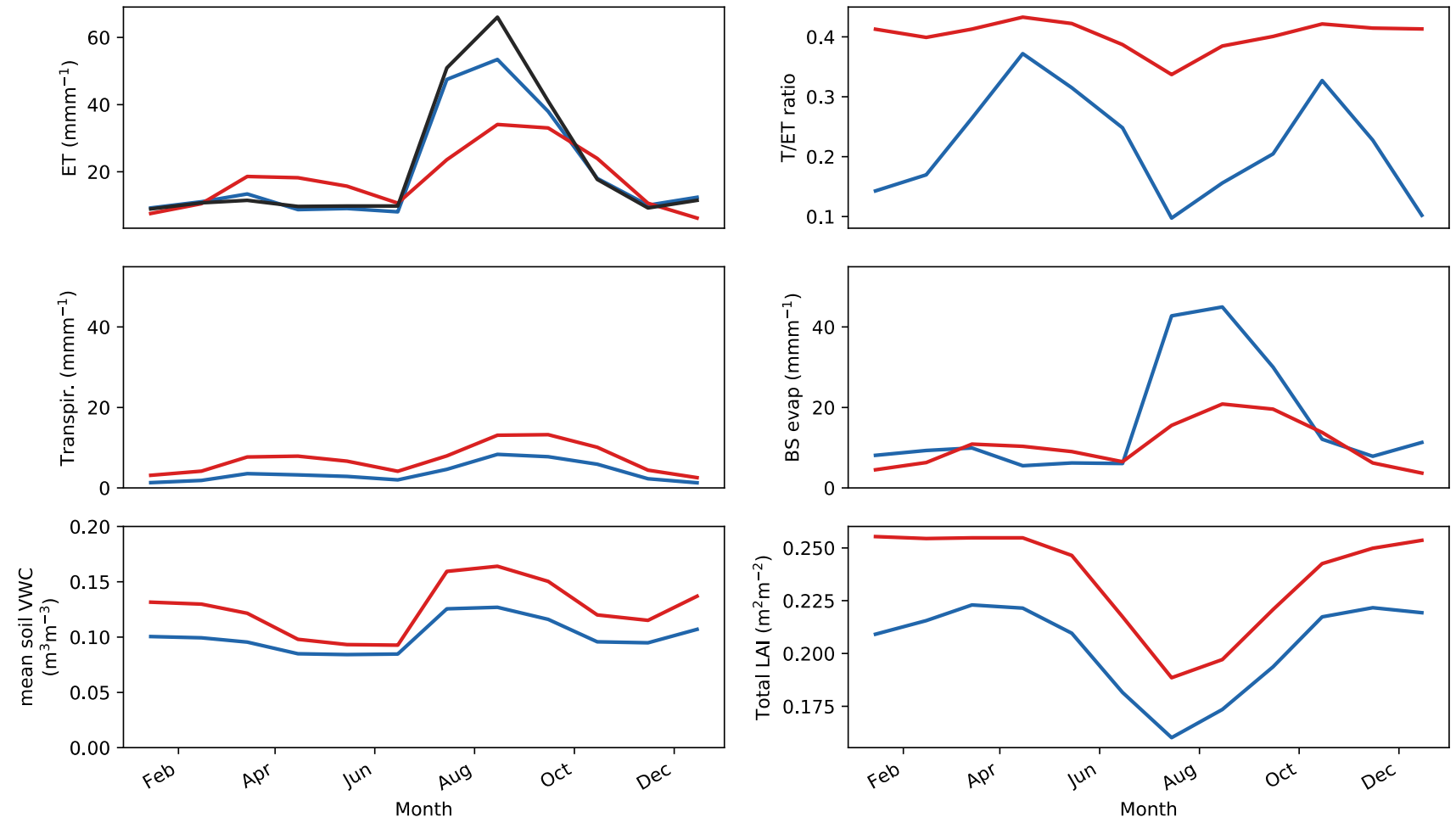

- 11 layer hydrol $\quad$ Bare soil evap resistance $\quad$ SITE DATA 


\section{e) US-SRG}

\section{US-SRG}
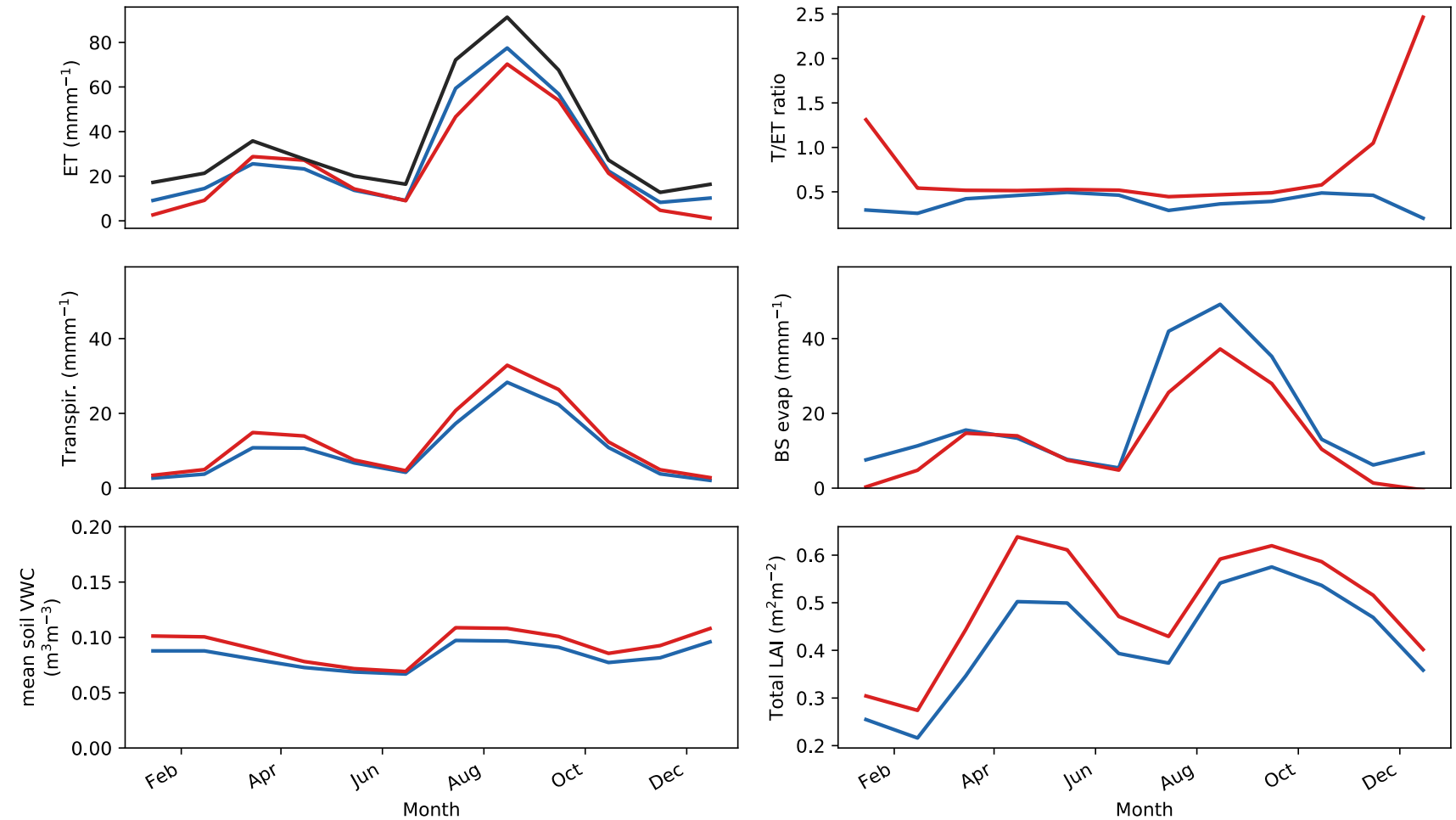

- 11 layer hydrol $\quad$ Bare soil evap resistance $\quad$ SITE DATA 


\section{f) US-Wkg}
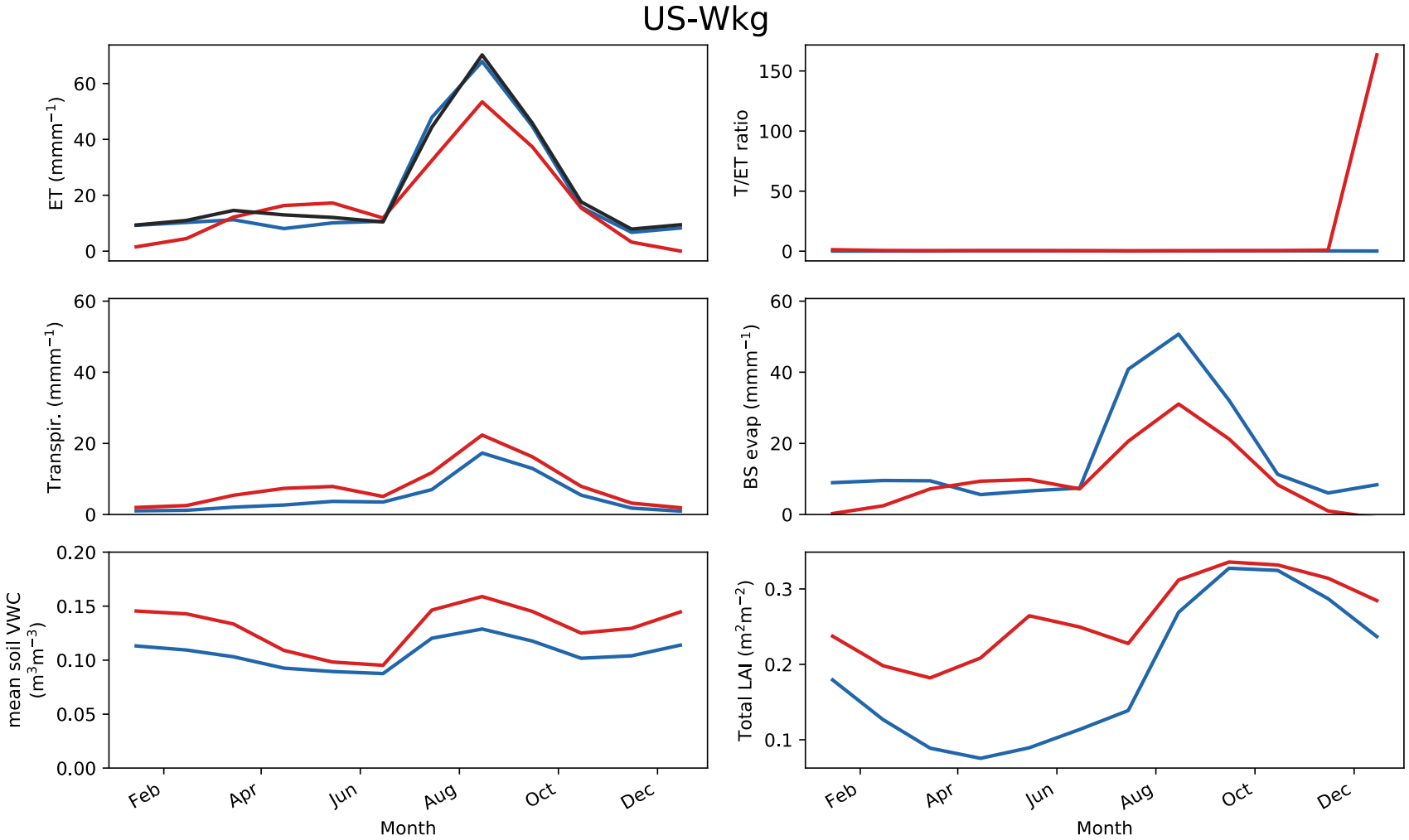

— 11 layer hydrol $\quad$ Bare soil evap resistance $\quad$ SITE DATA

485 
Figure S10: Monthly mean seasonal cycle for all sites comparing the default 11LAY simulations (blue curve) with a) with a simulation 500 that increased the $\mathbf{C 4}$ grass fraction at the expense of the bare soil fraction (yellow curve); and b) a simulation that included an additional bare soil evaporation resistance term (red curve). In all subfigures - top left: mean soil moisture; top right: ET compared to observation (black curve); bottom left: transpiration; bottom right: bare soil evaporation. Units in mmmonth-1.

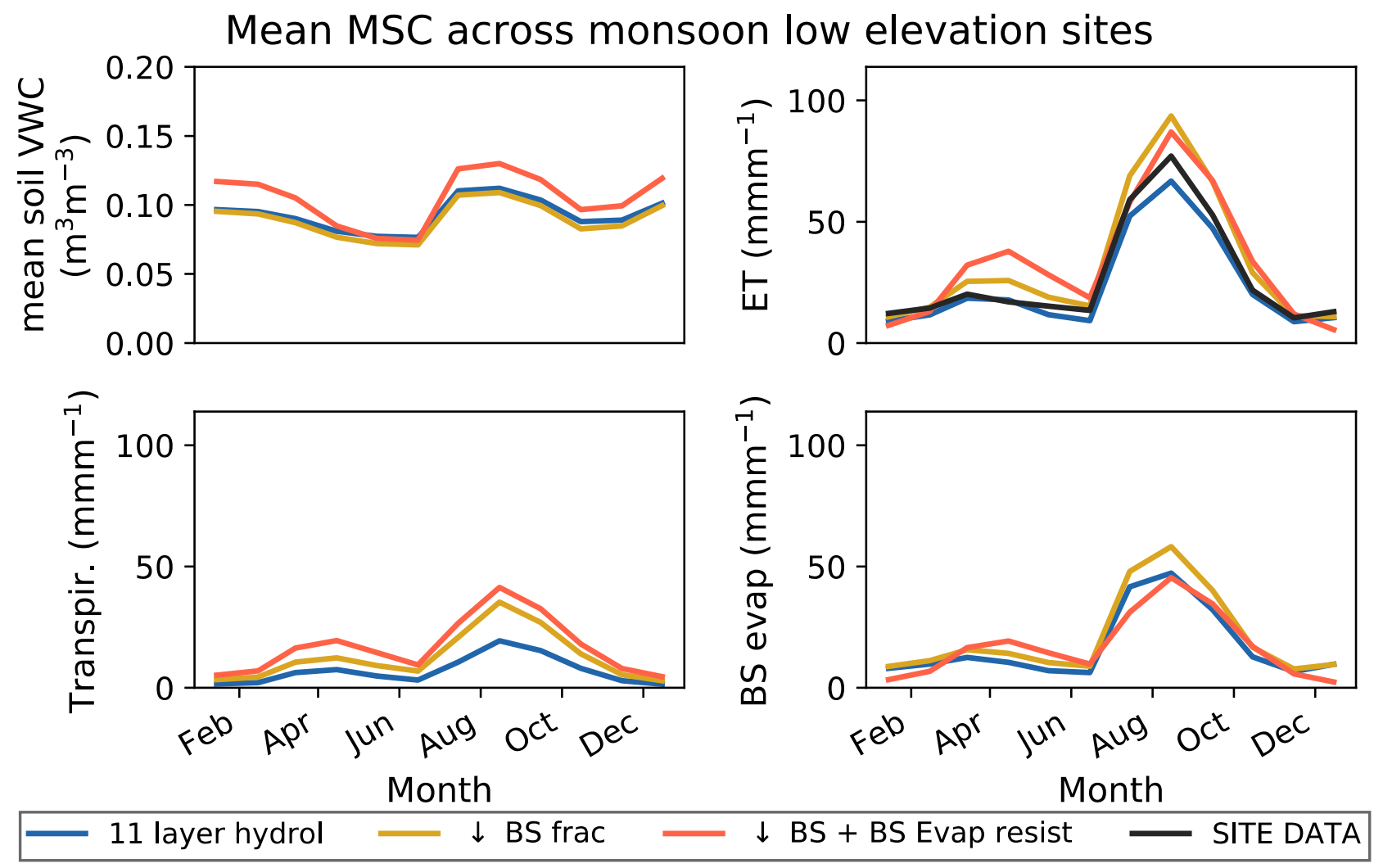

